Step 1: Genetic variants associated

with both body fat $\%$ and composite metabolic biomarkers

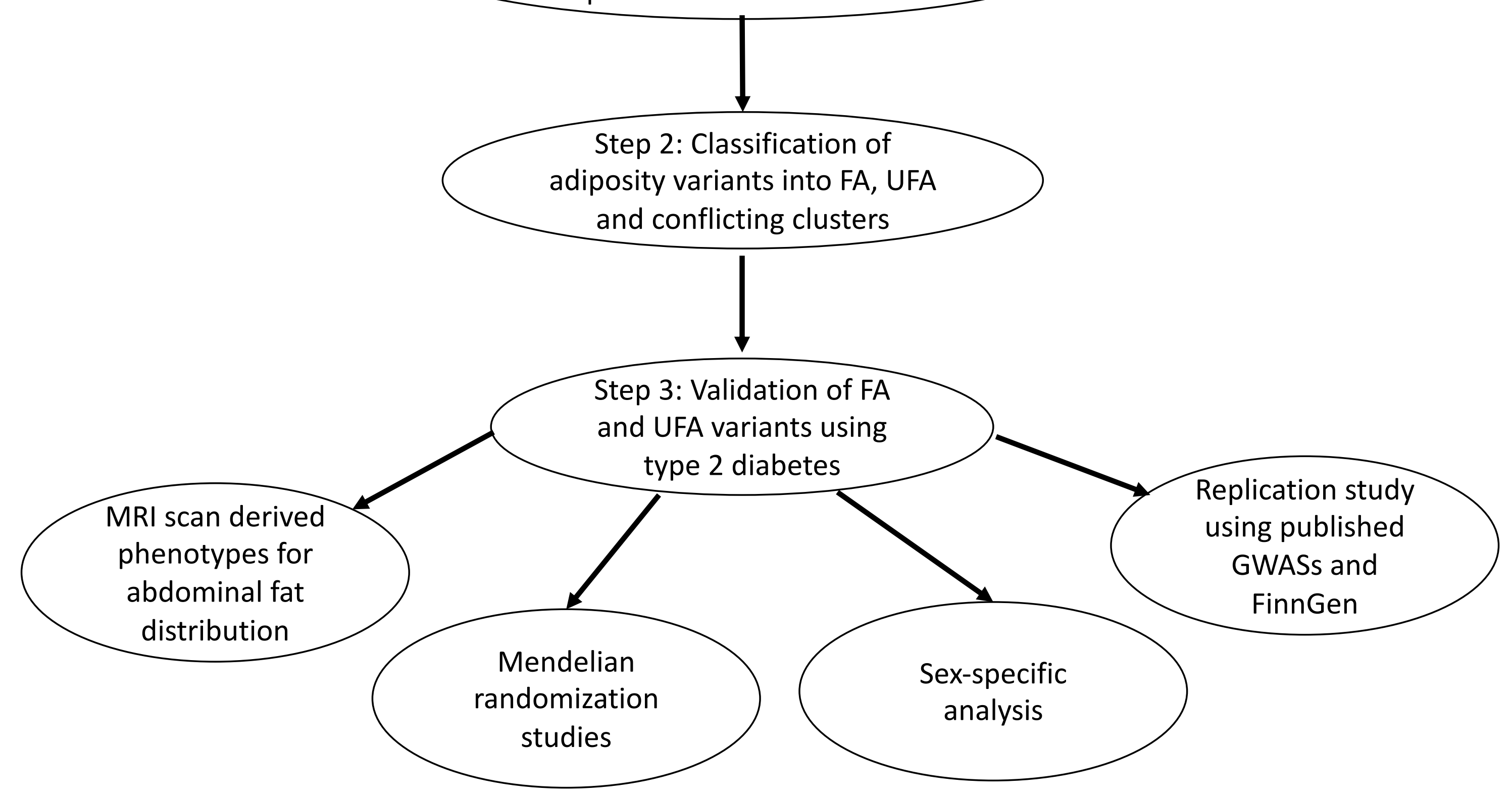


"Favourable" adiposity

36

variants
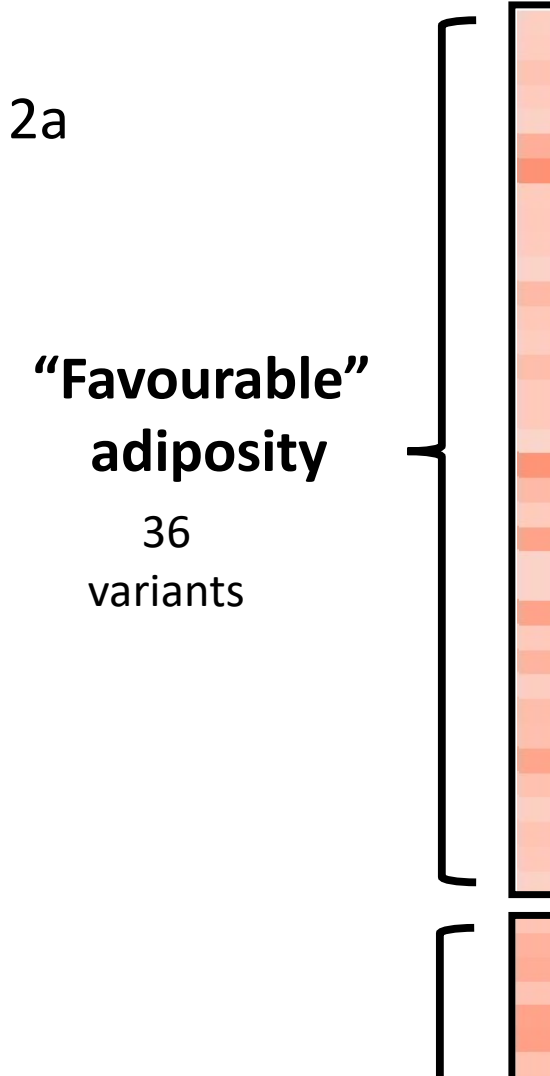

"Unfavourable" adiposity

38

variants

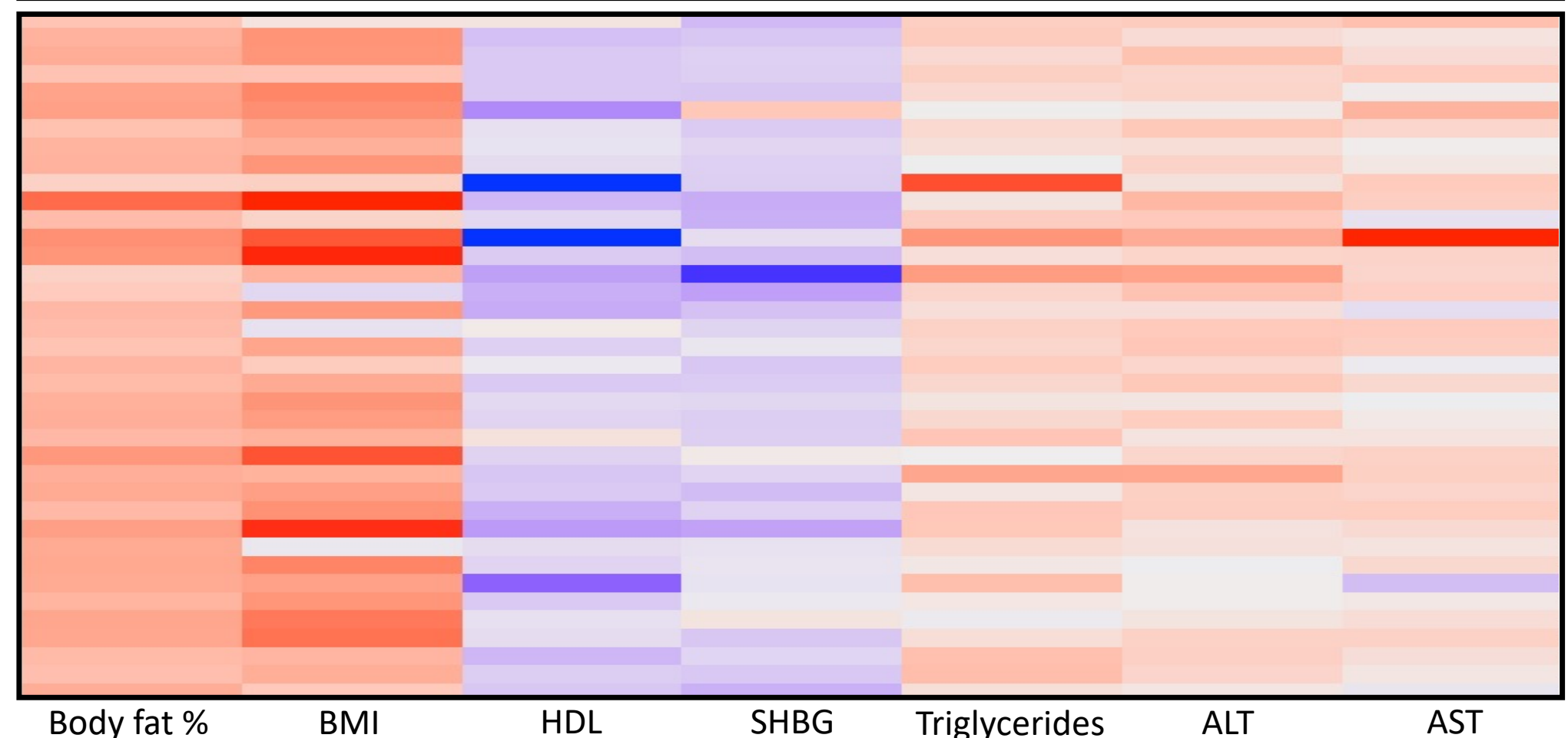




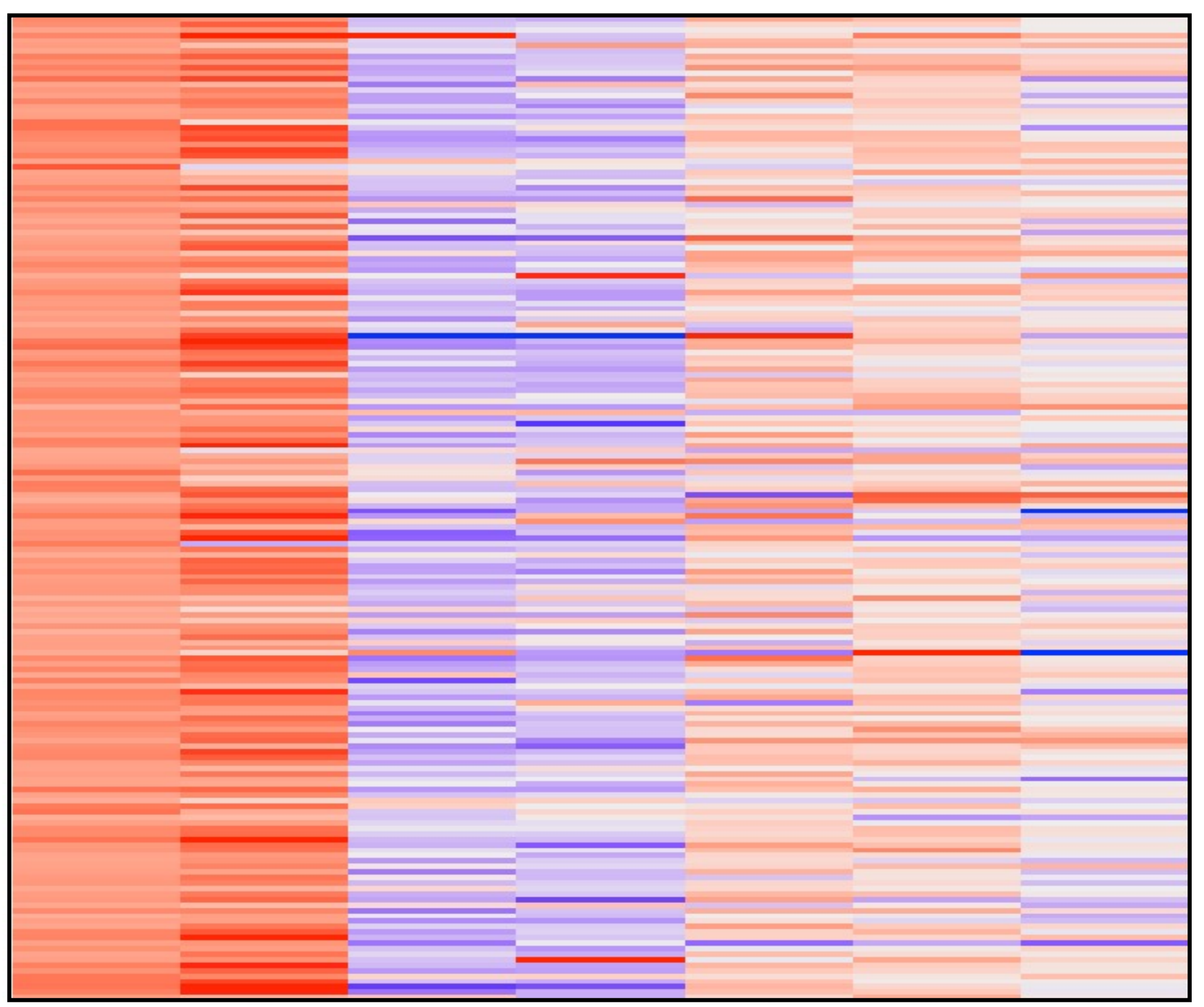

Conflicting cluster

180

variants

: 
SF $3 a$

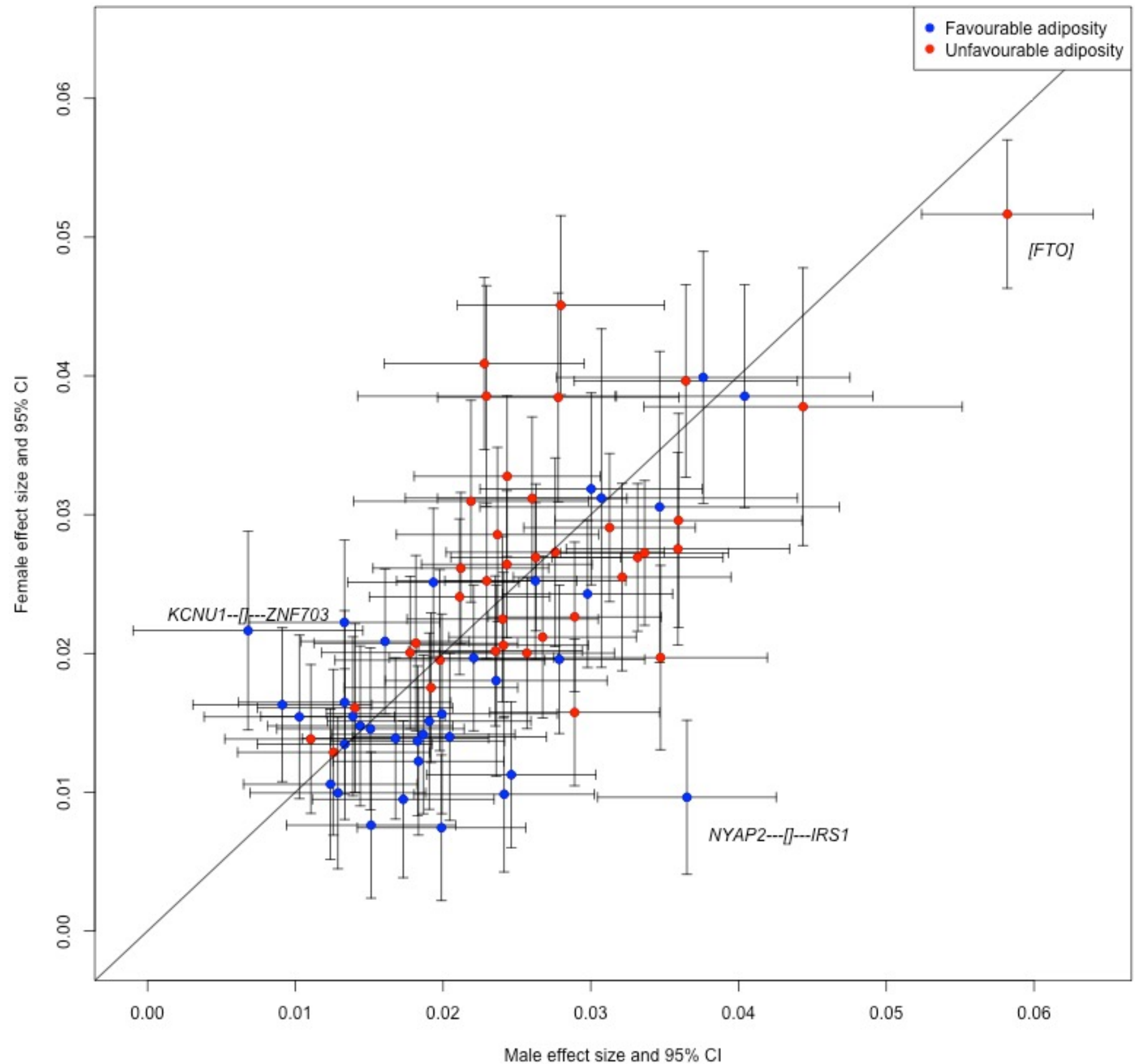




\section{SF 3b}

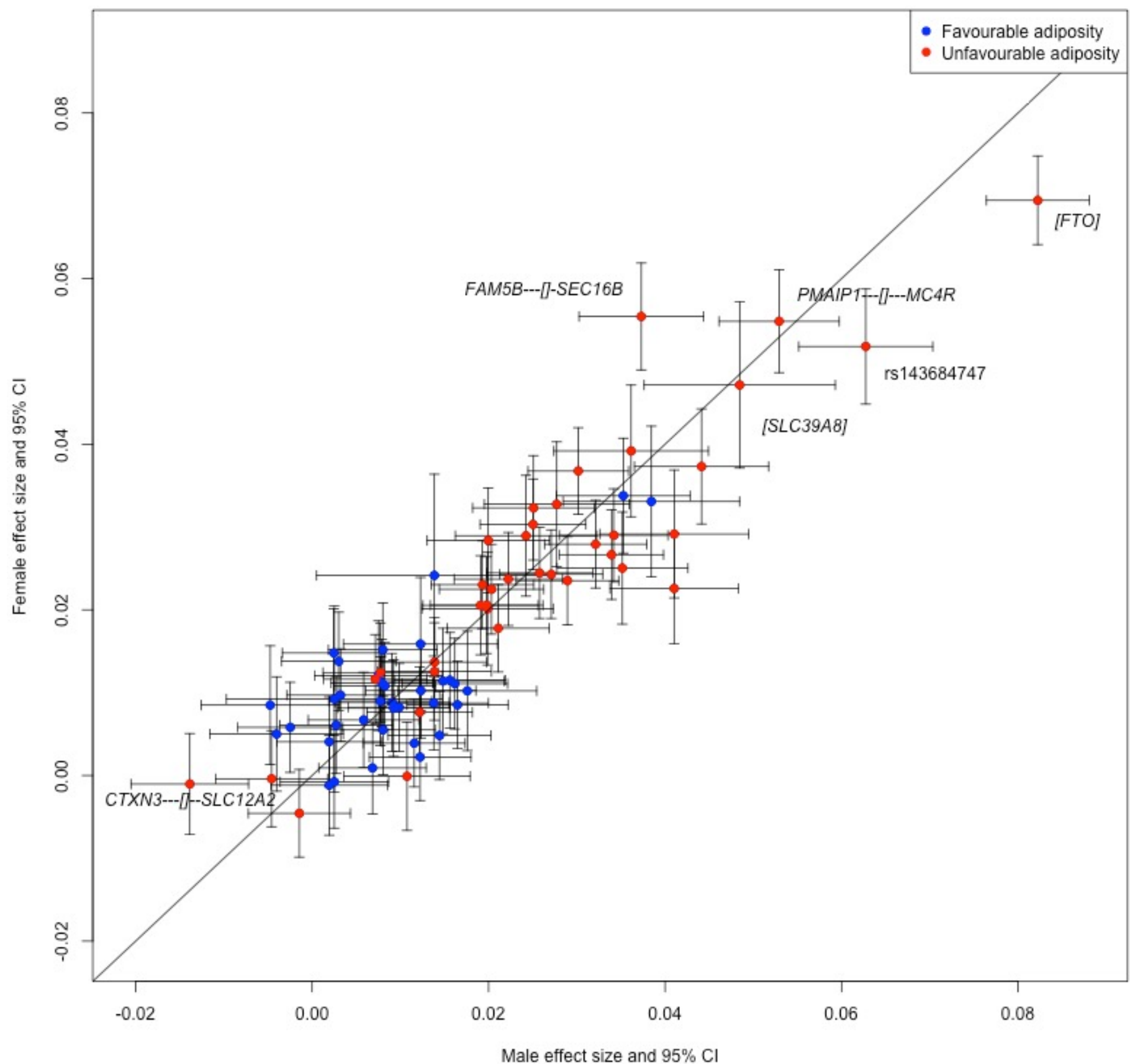


SF $3 c$

HDL

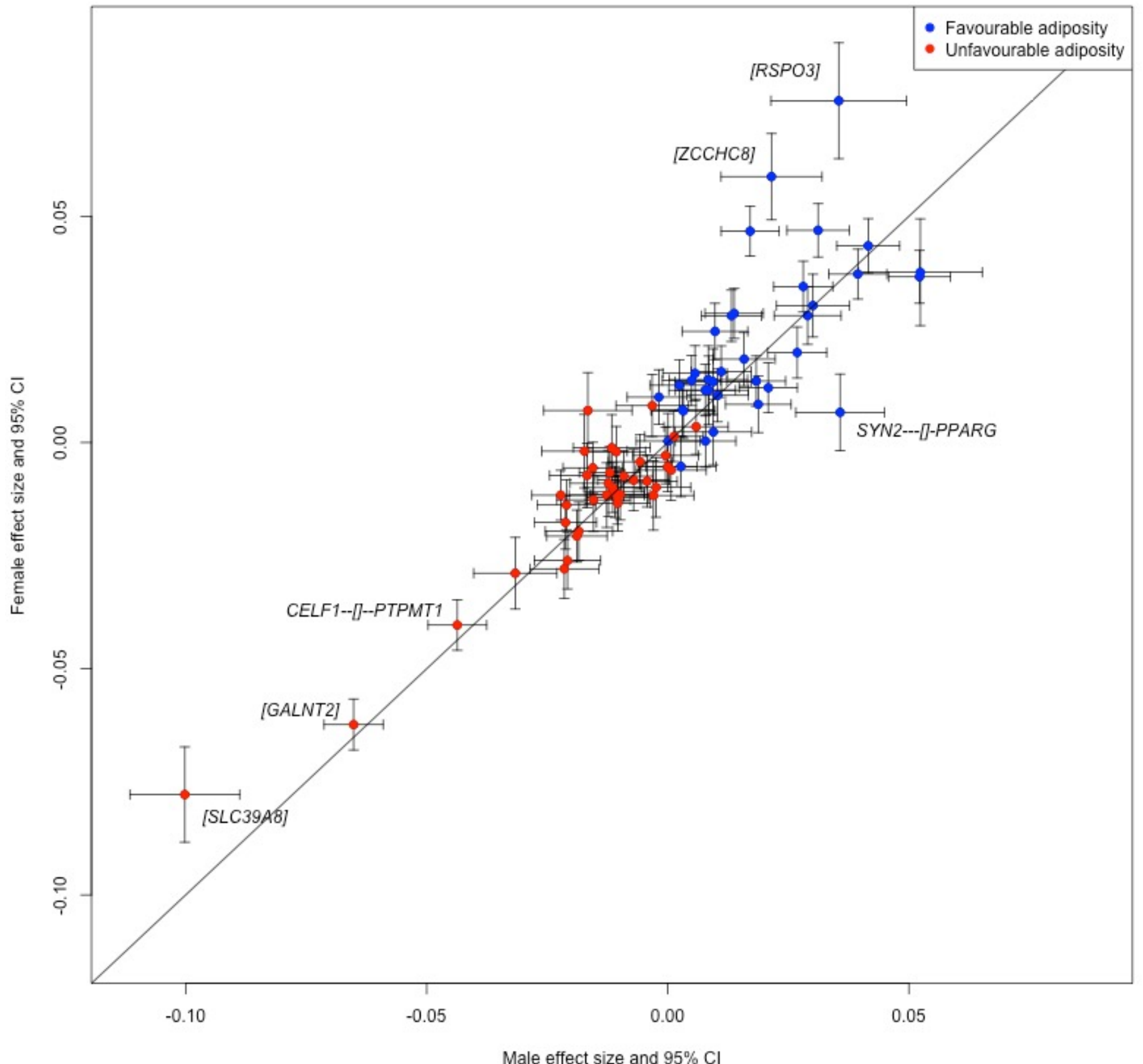


SF 3d

SHBG

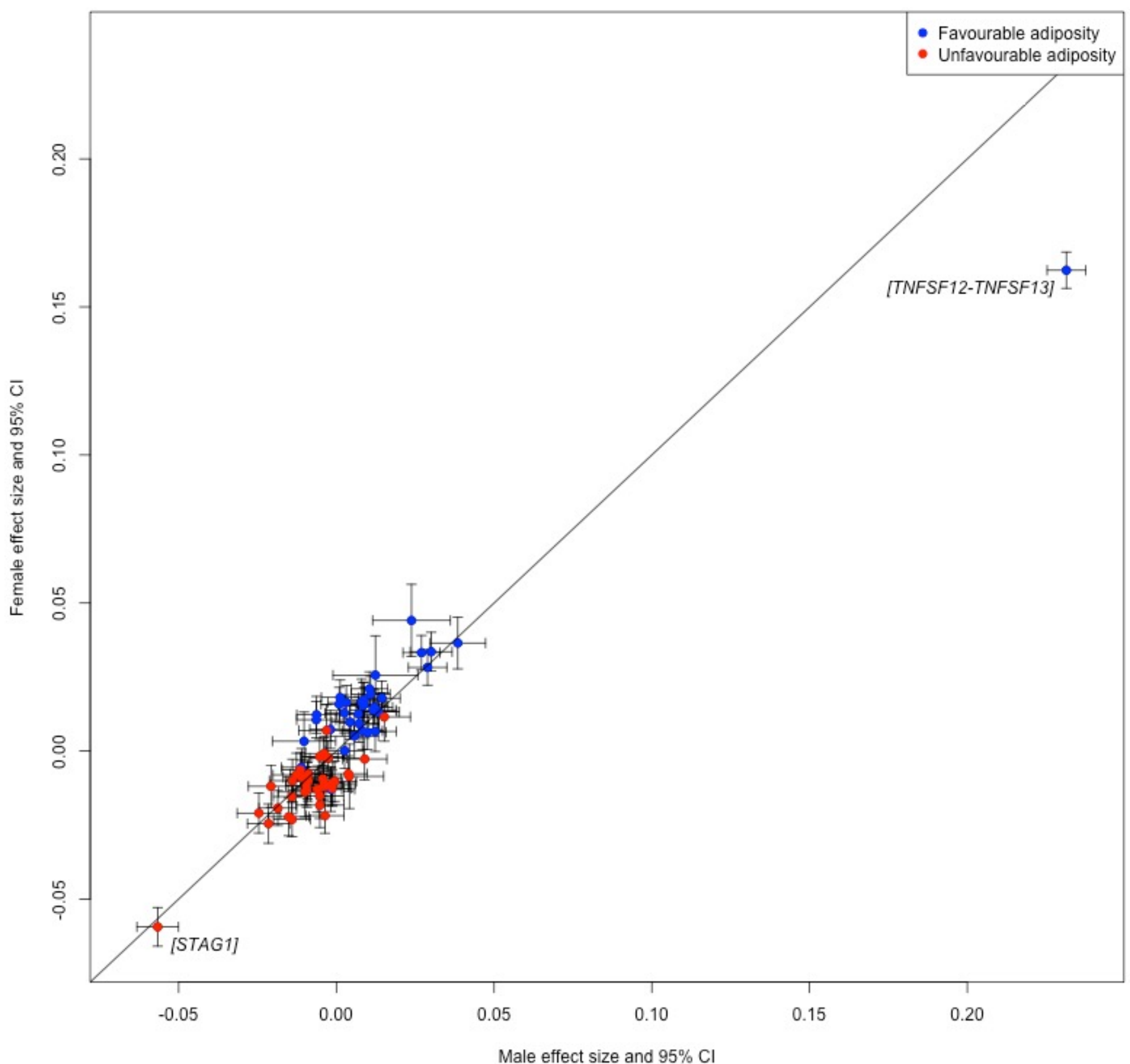


SF $3 e$

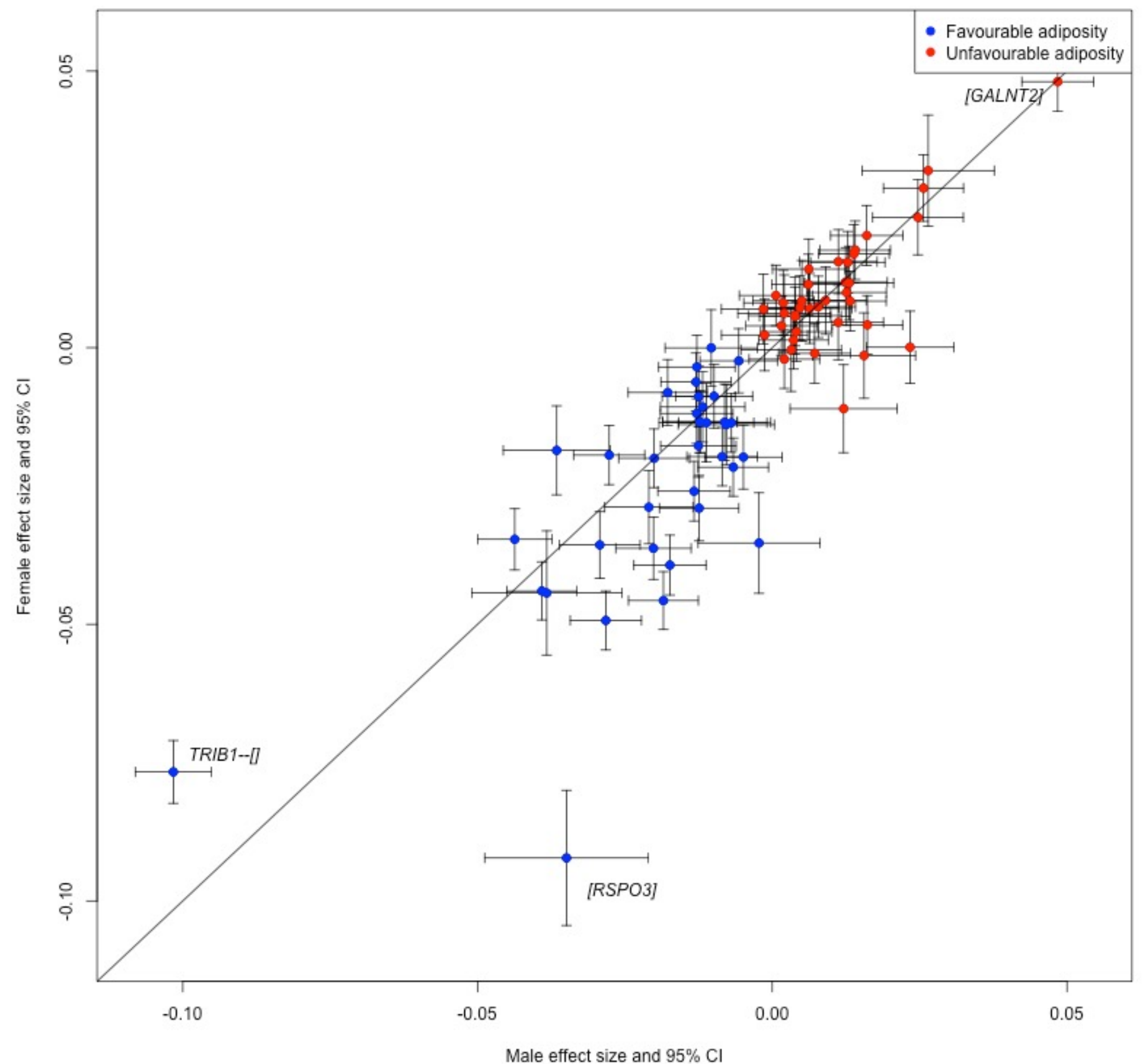




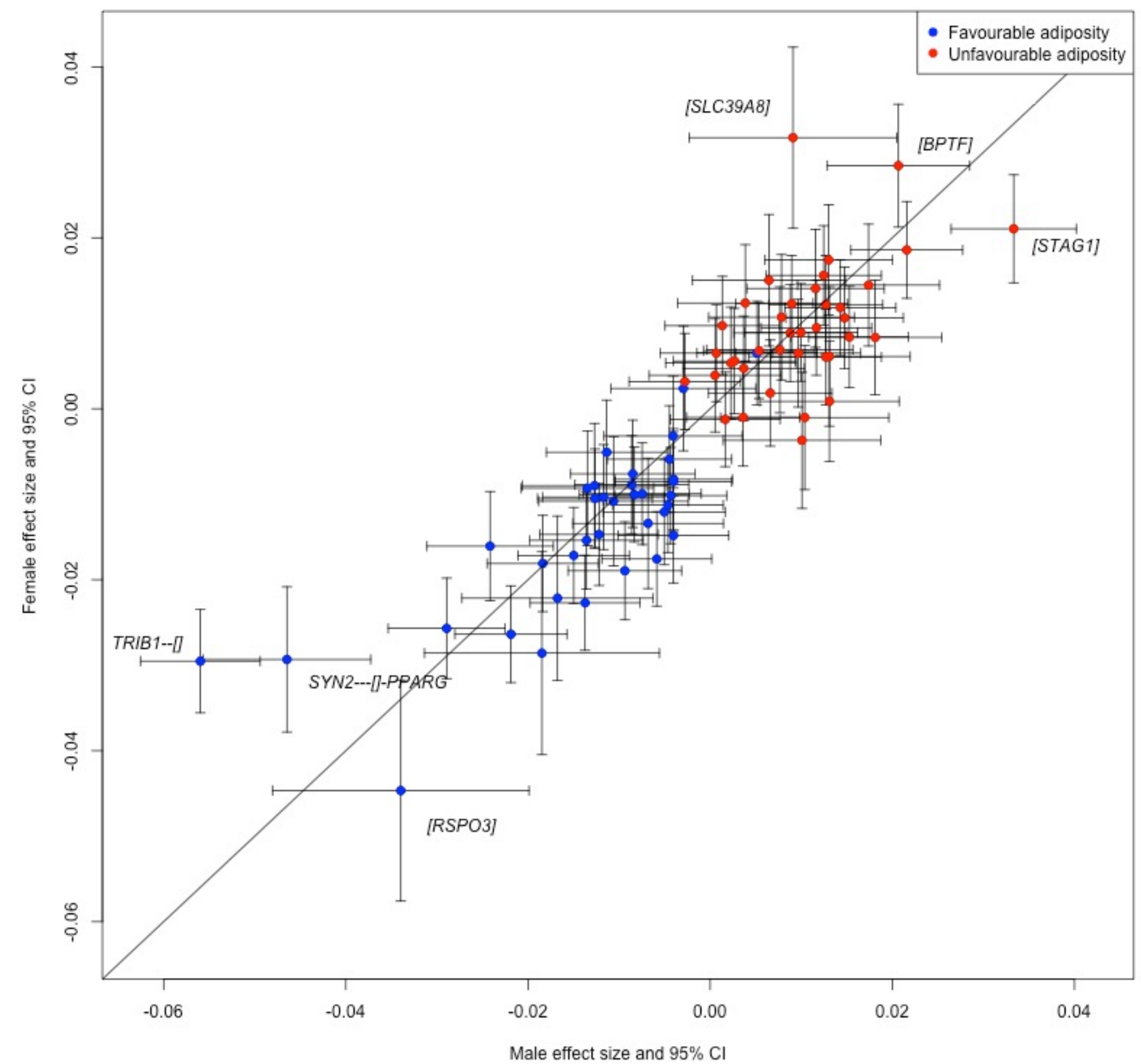




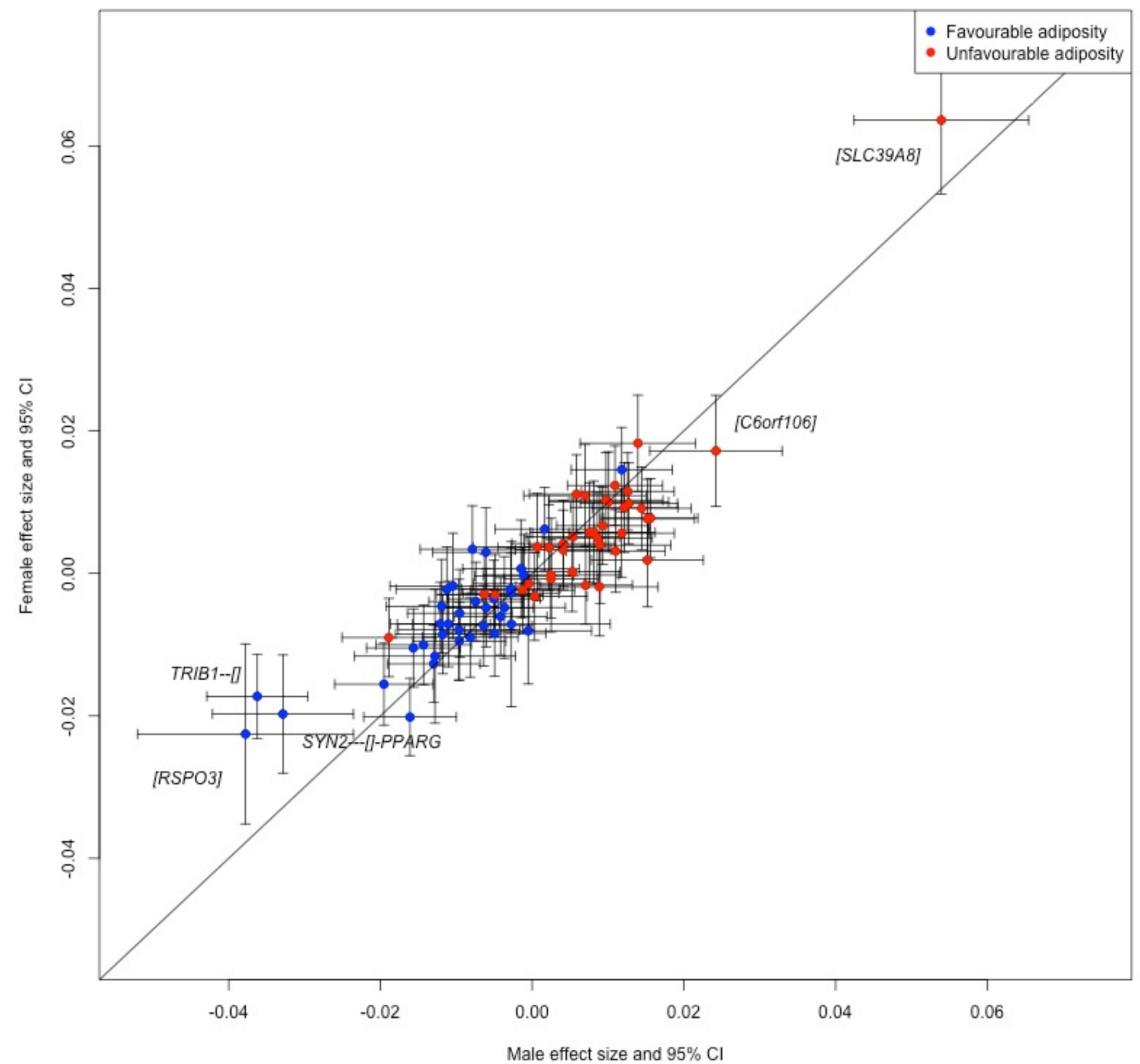




\section{SF $3 h$}

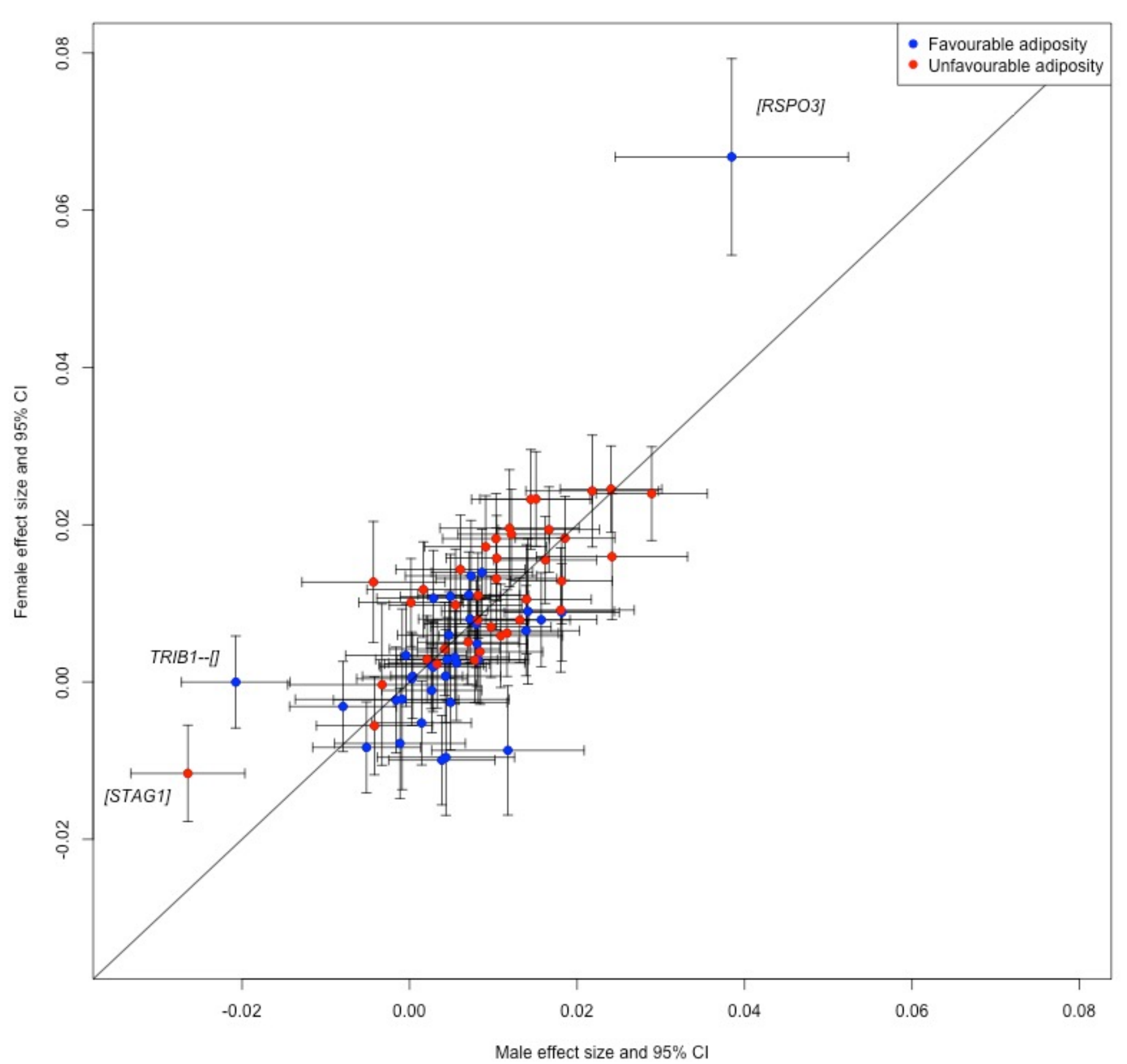


SF 3i

ASAT

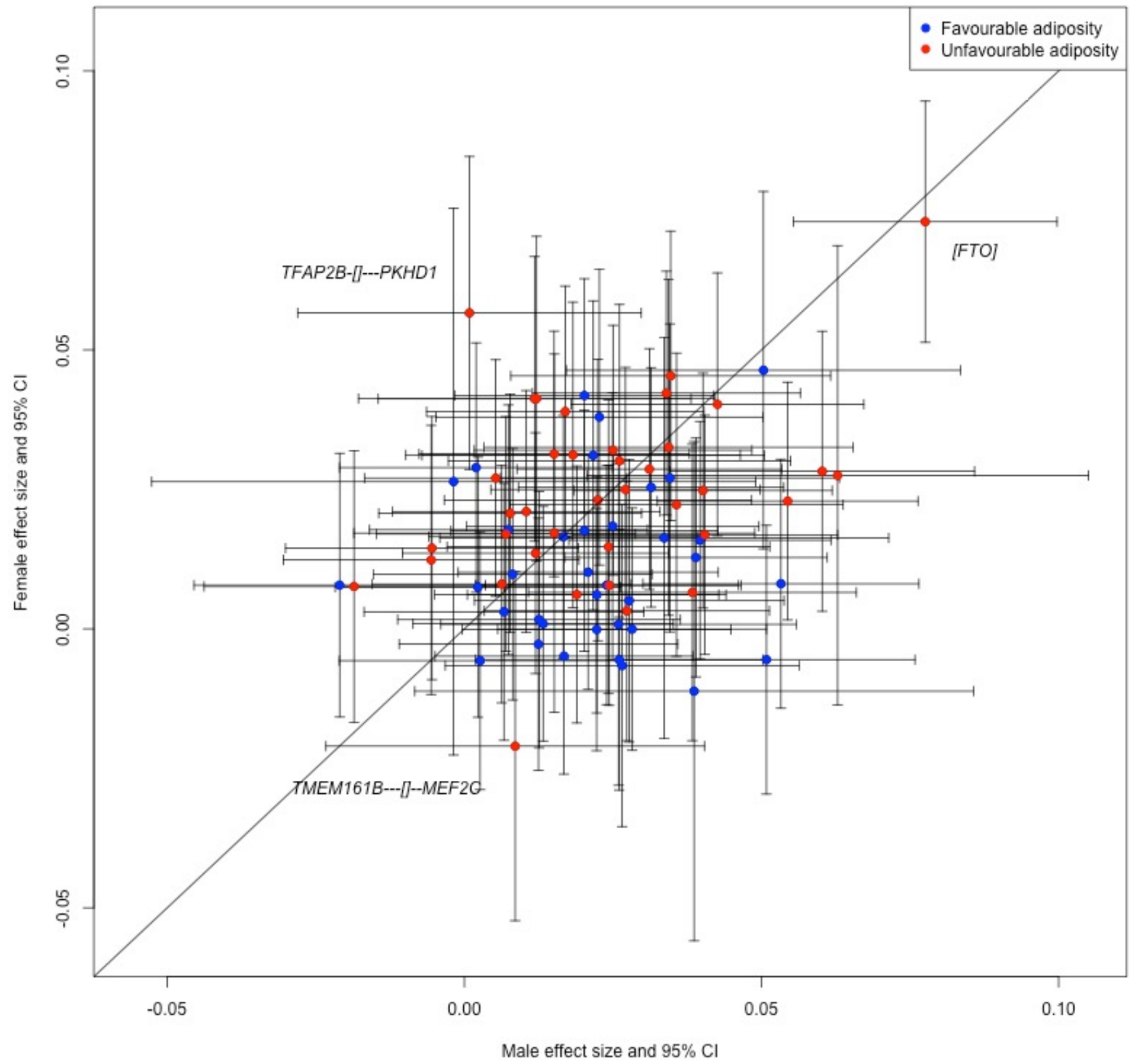




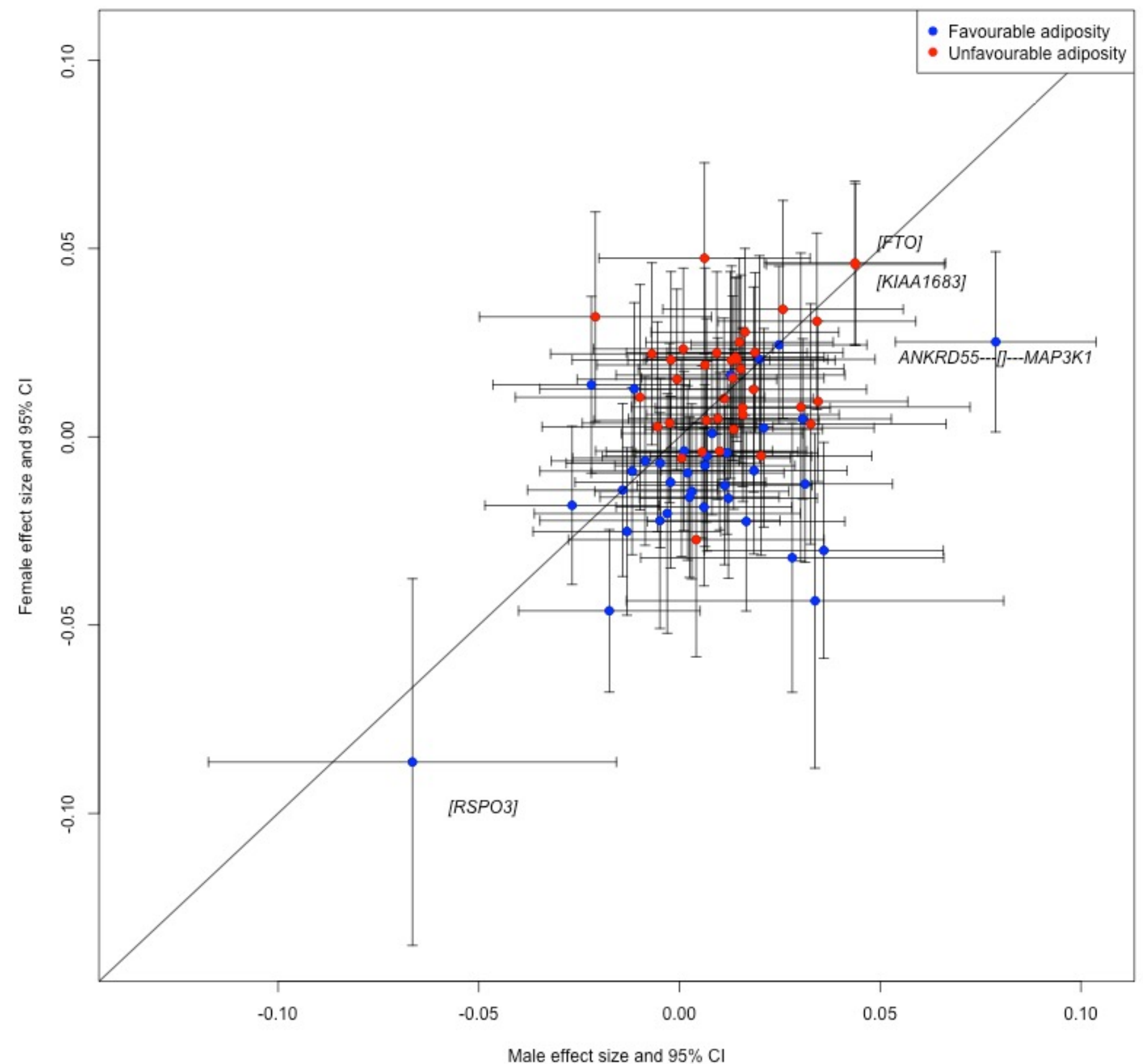


SF 3k

VATSAT

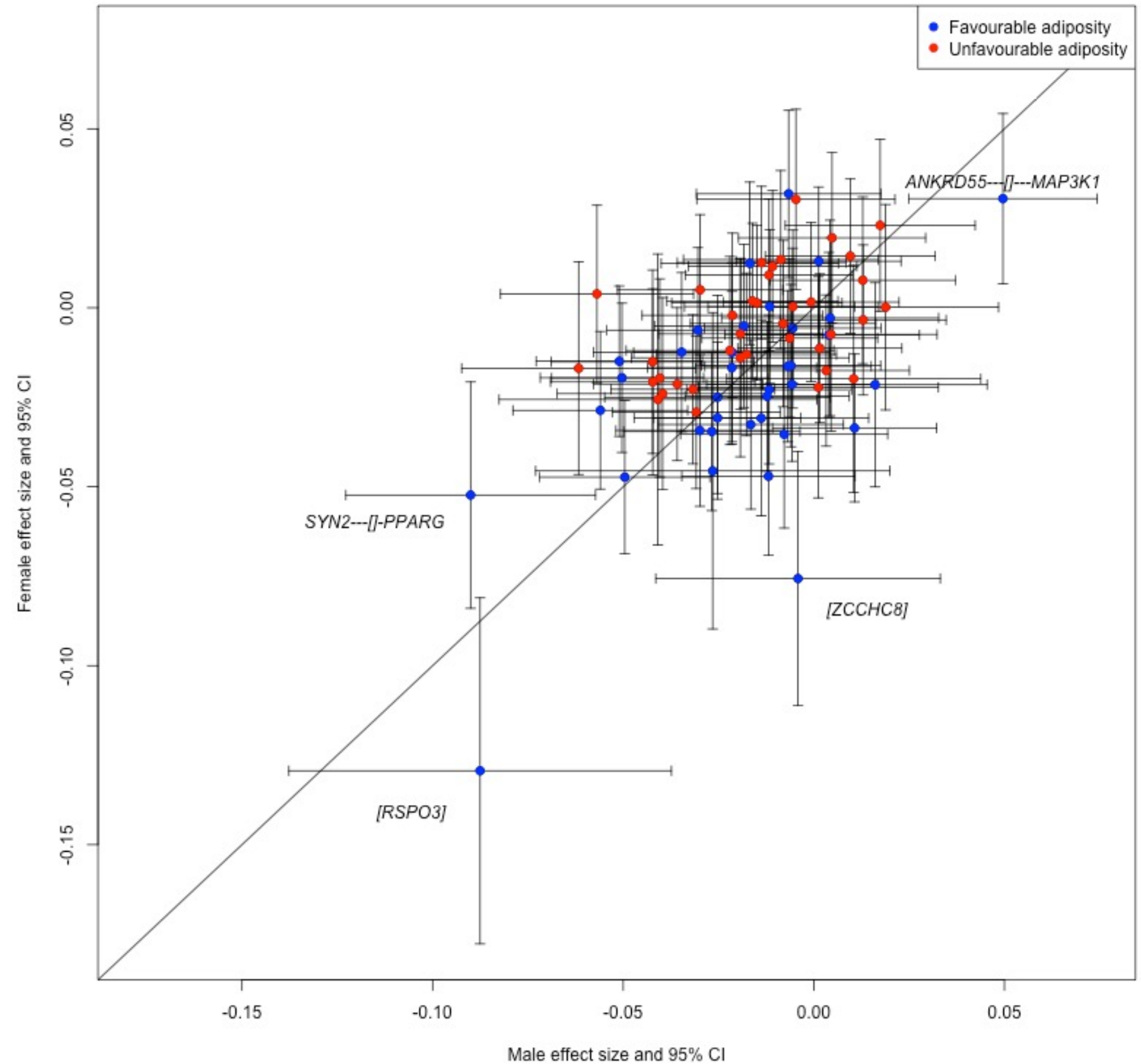


SF 31

Liver volume

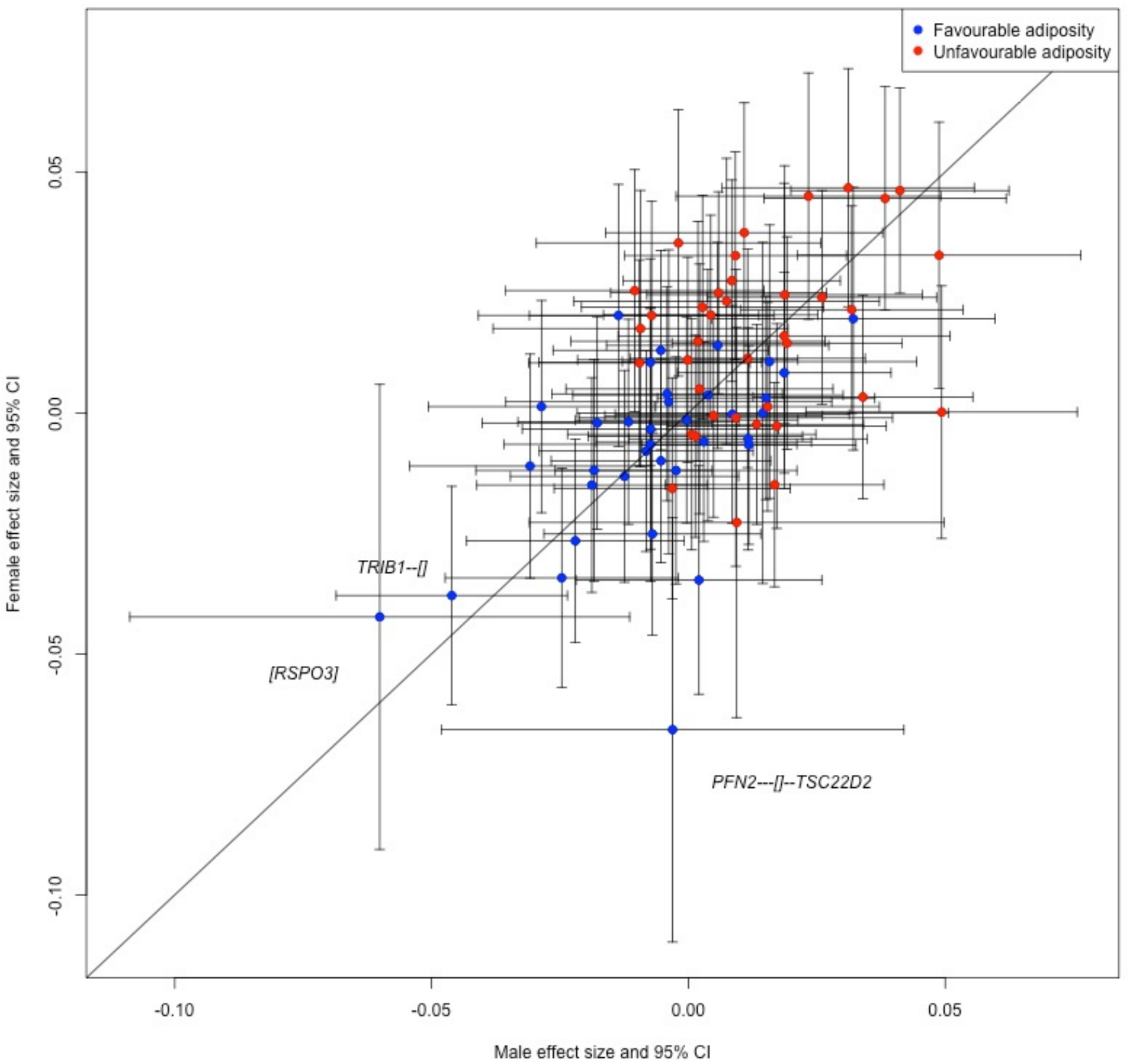


SF $3 \mathrm{~m}$

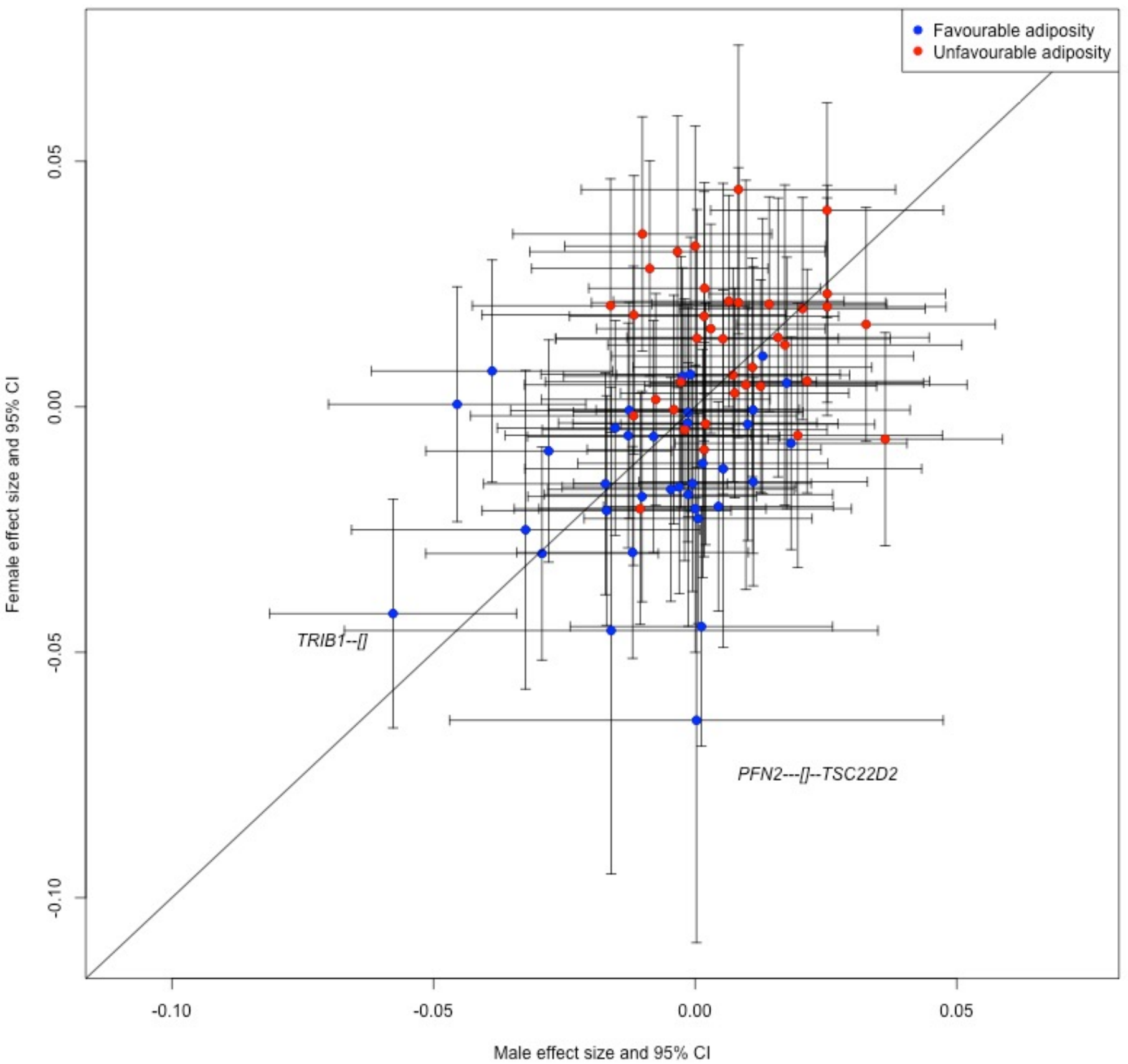


SF 3n

Pancreas volume

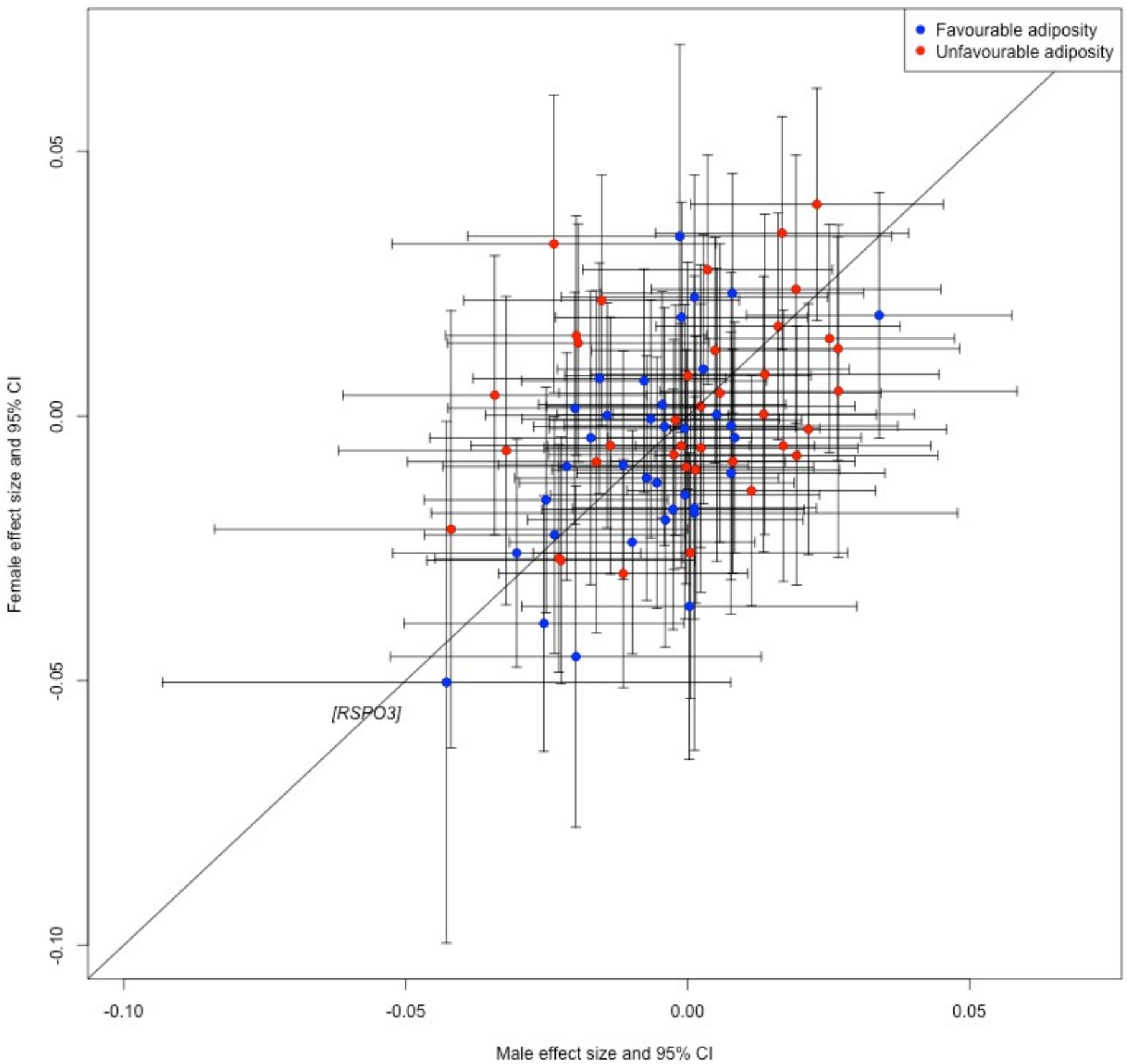


SF 30

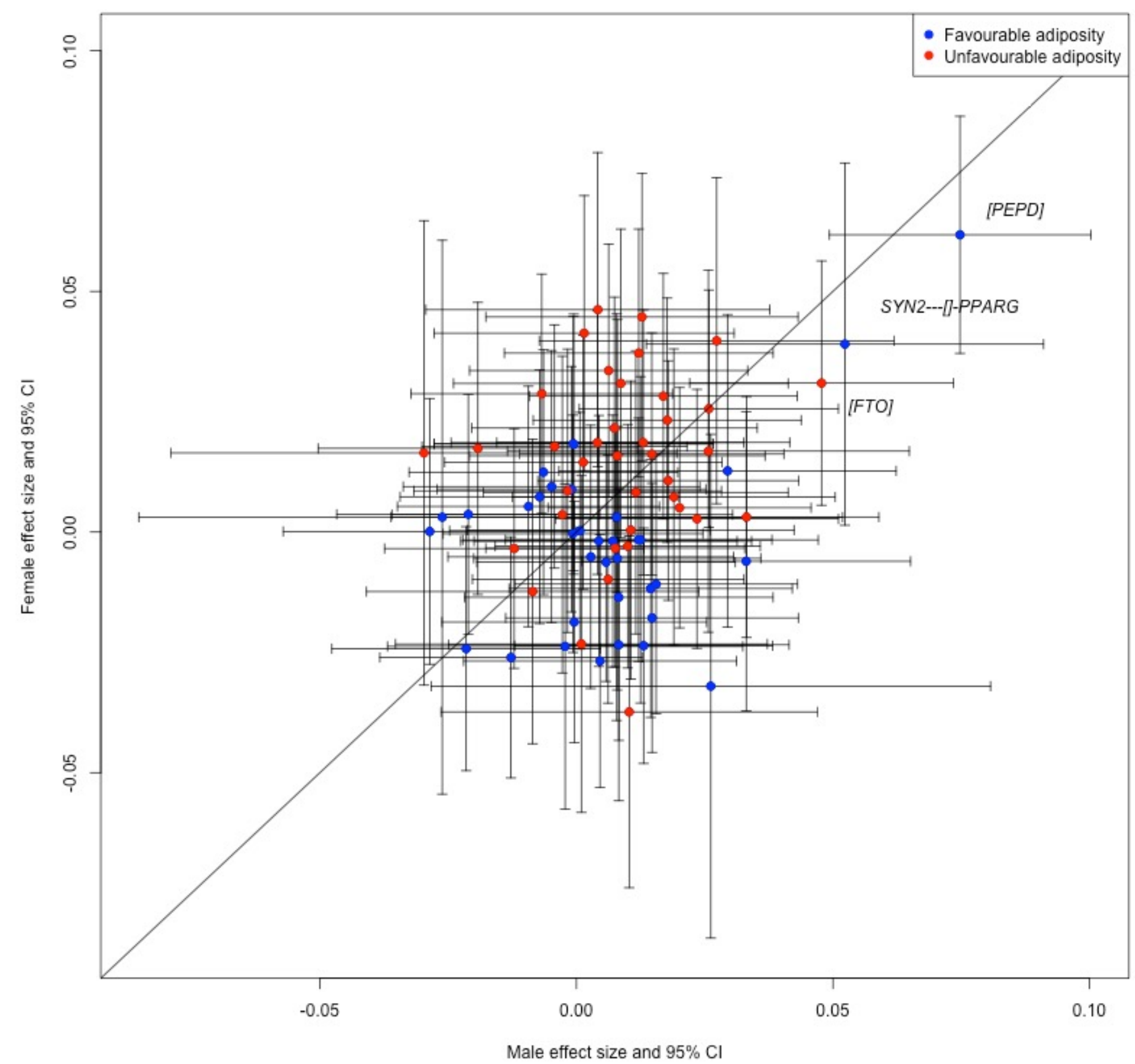




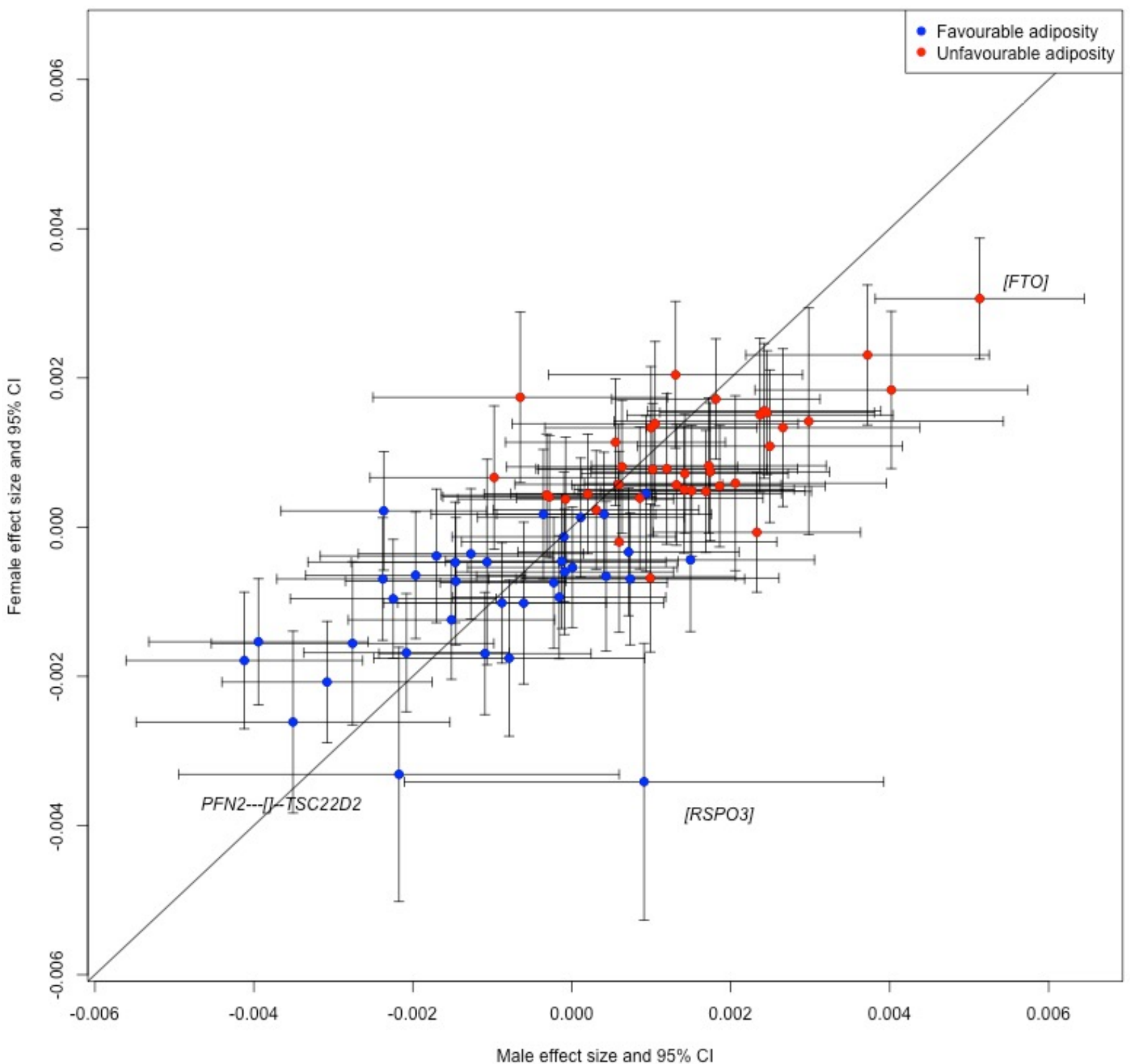




\section{SF $3 q$}

Heart disease

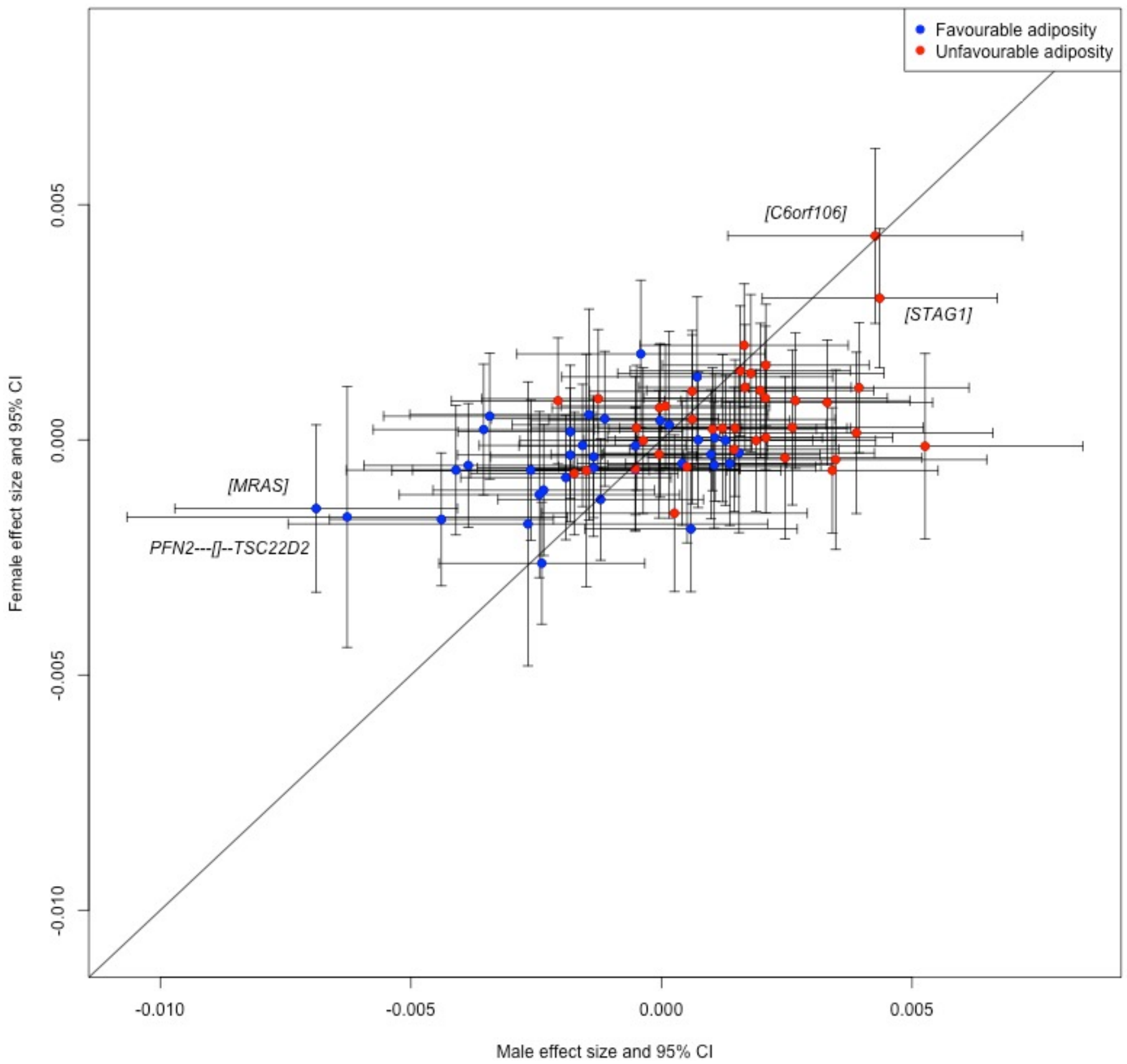




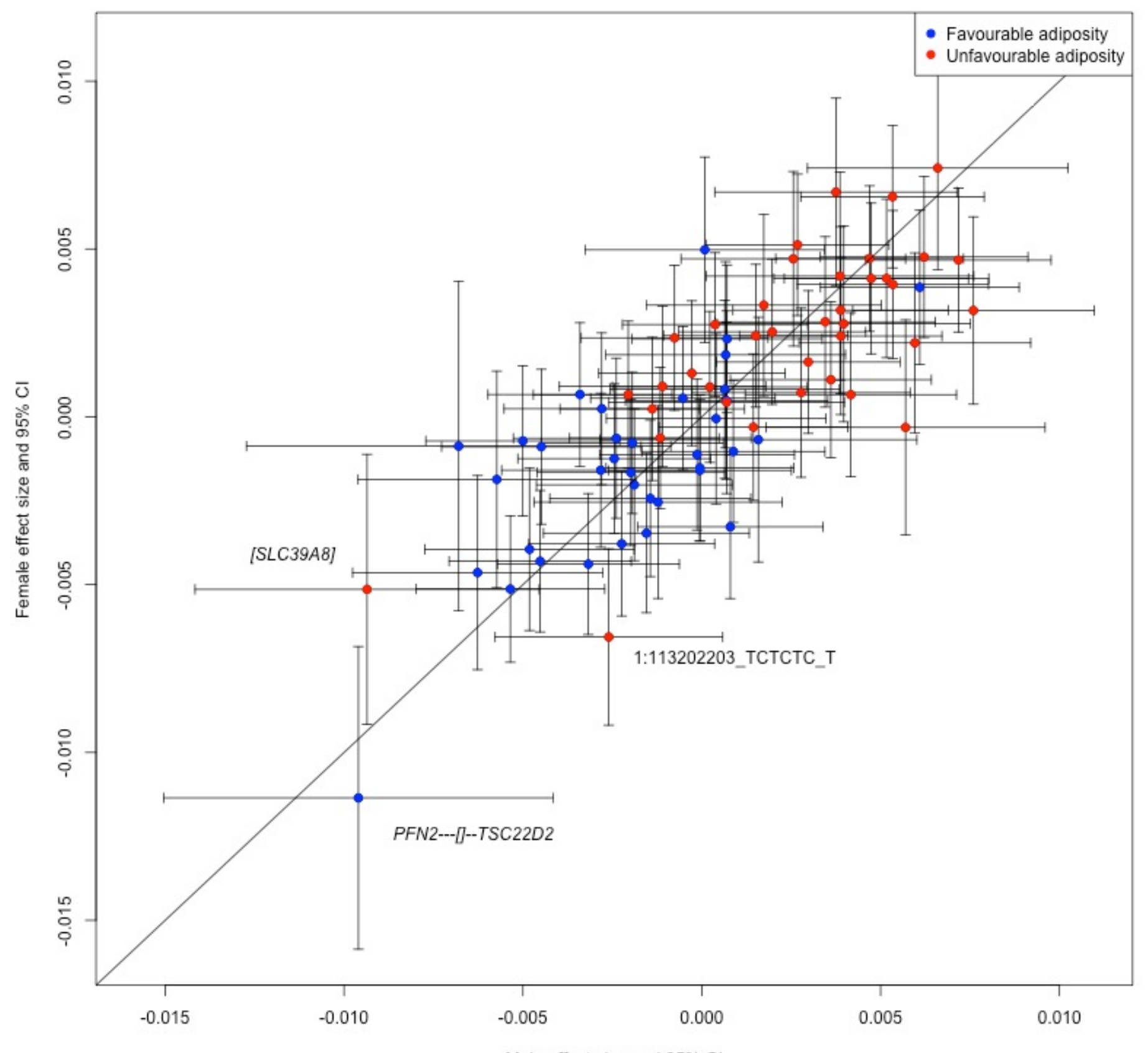

Male effect size and $95 \% \mathrm{Cl}$ 
SF 3s

Stroke

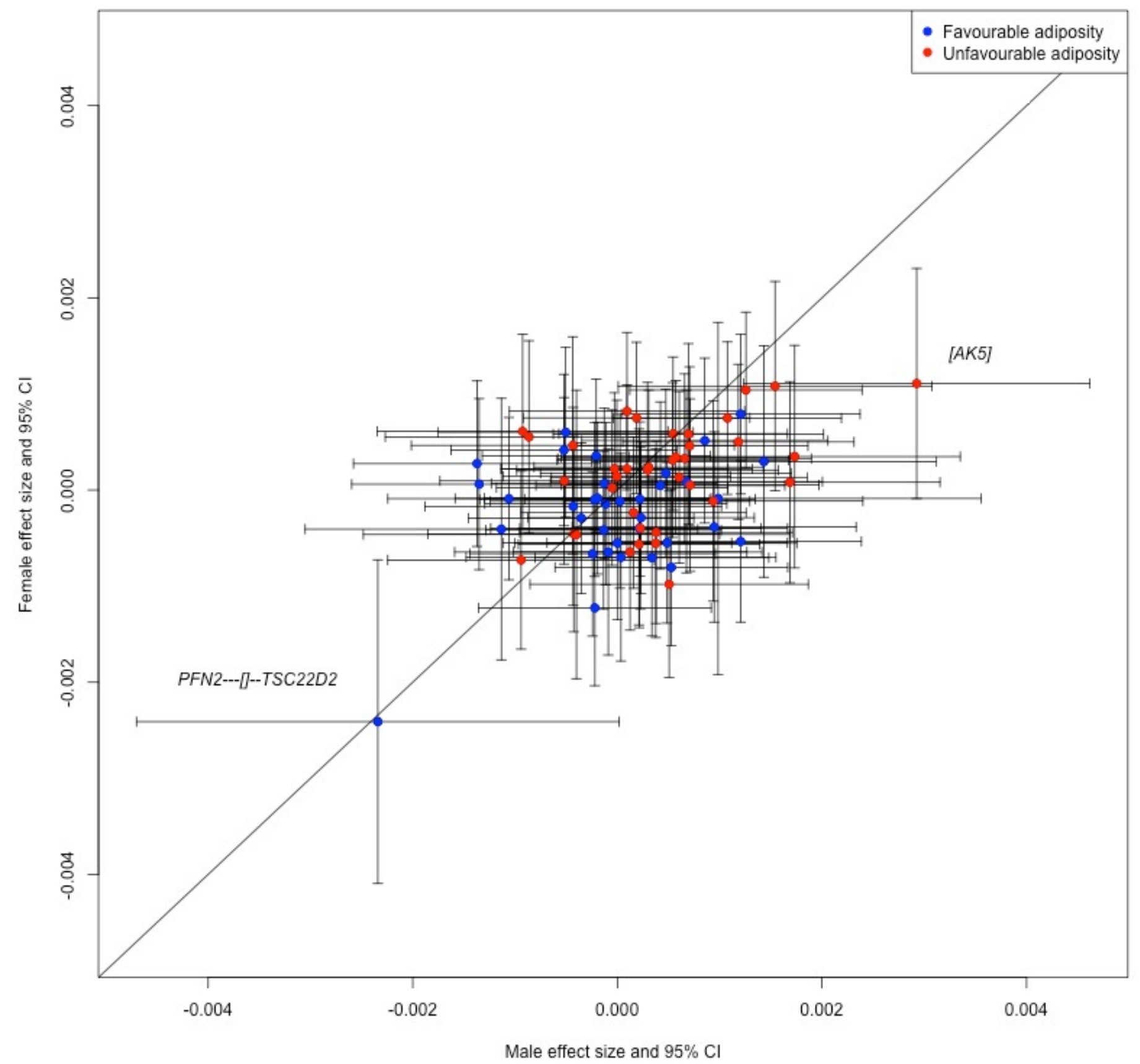


SF $3 t$

NAFLD

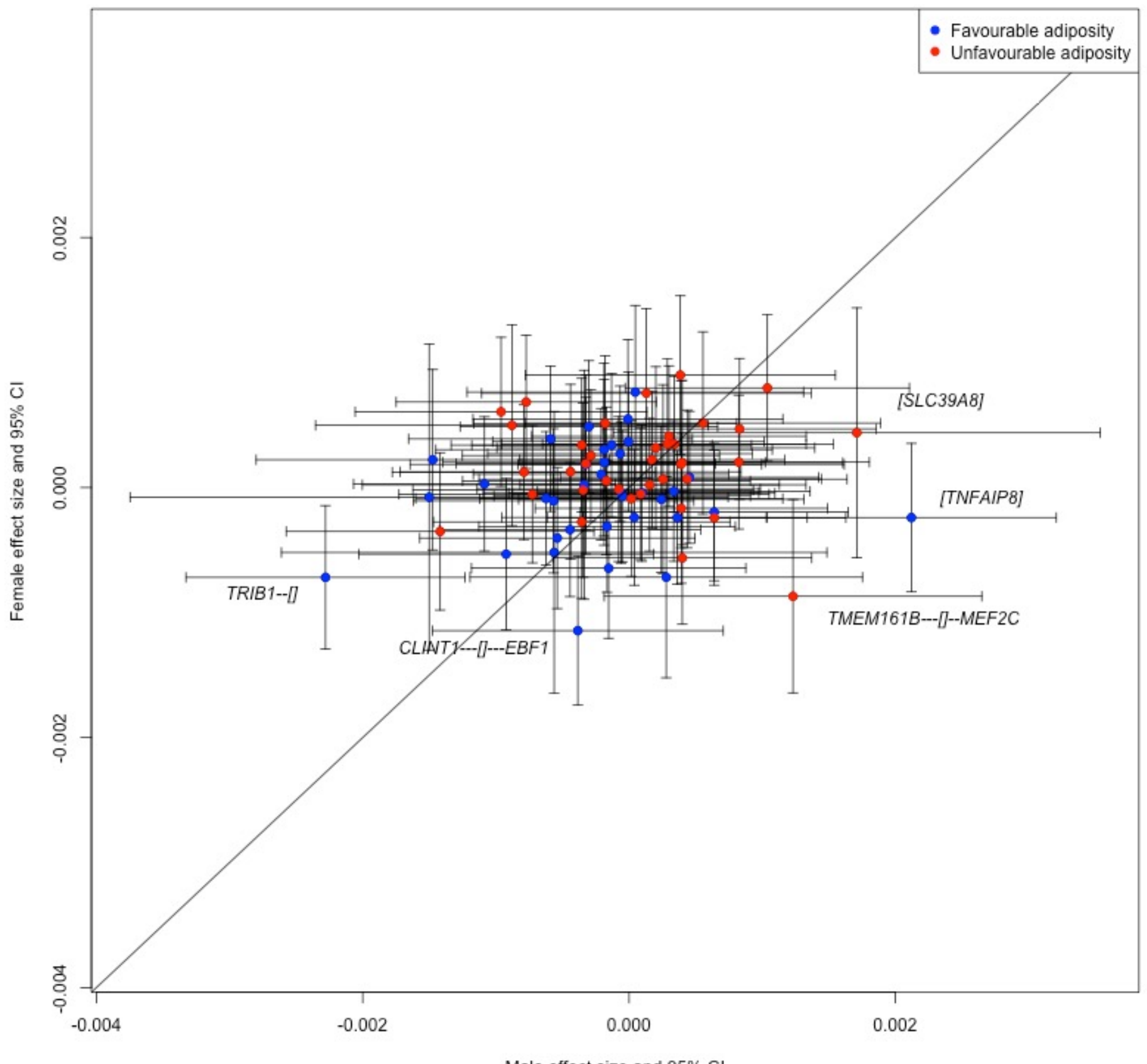

Male effect size and $95 \% \mathrm{Cl}$ 


\section{Favourable adiposity}

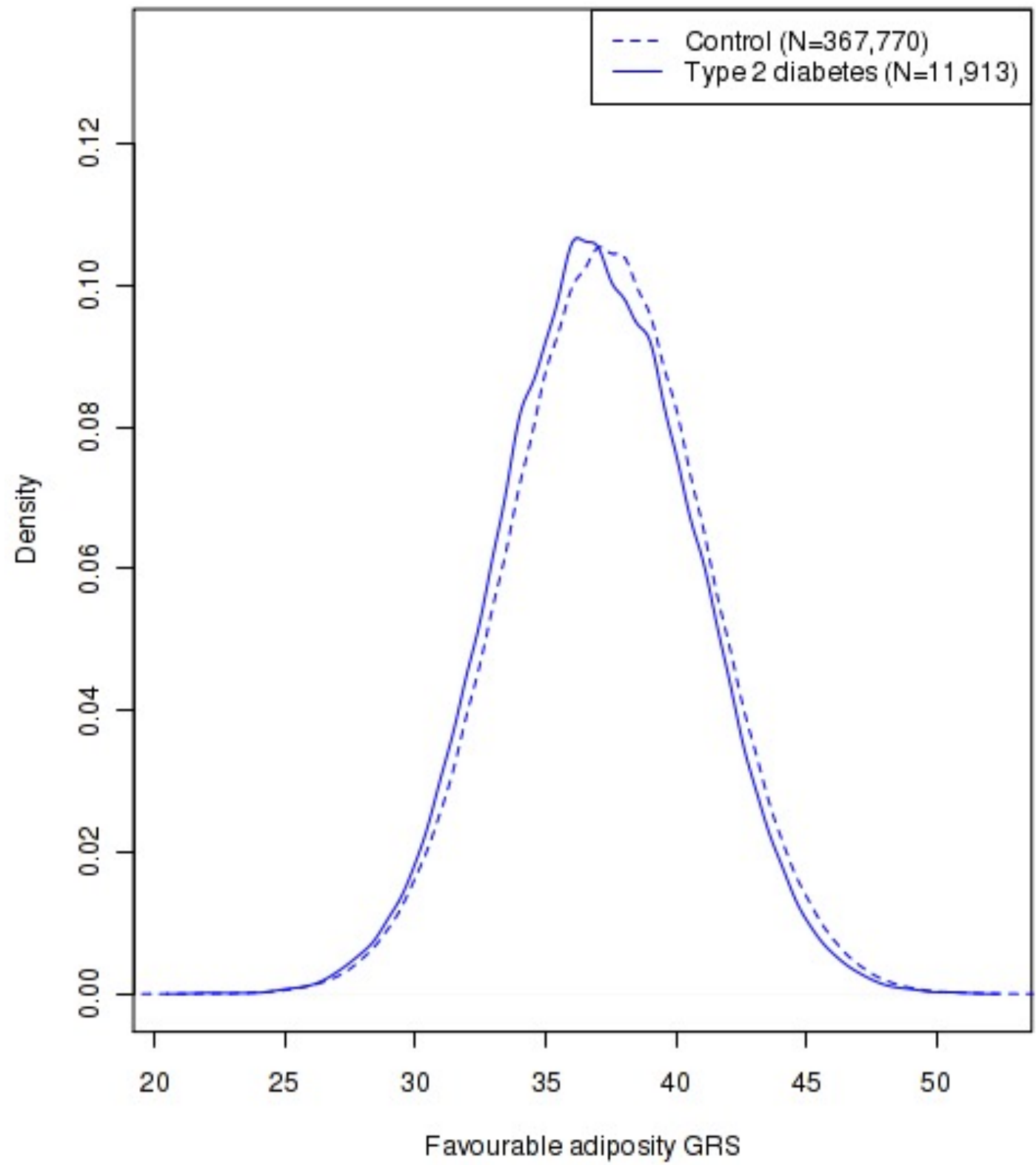

Unfavourable adiposity

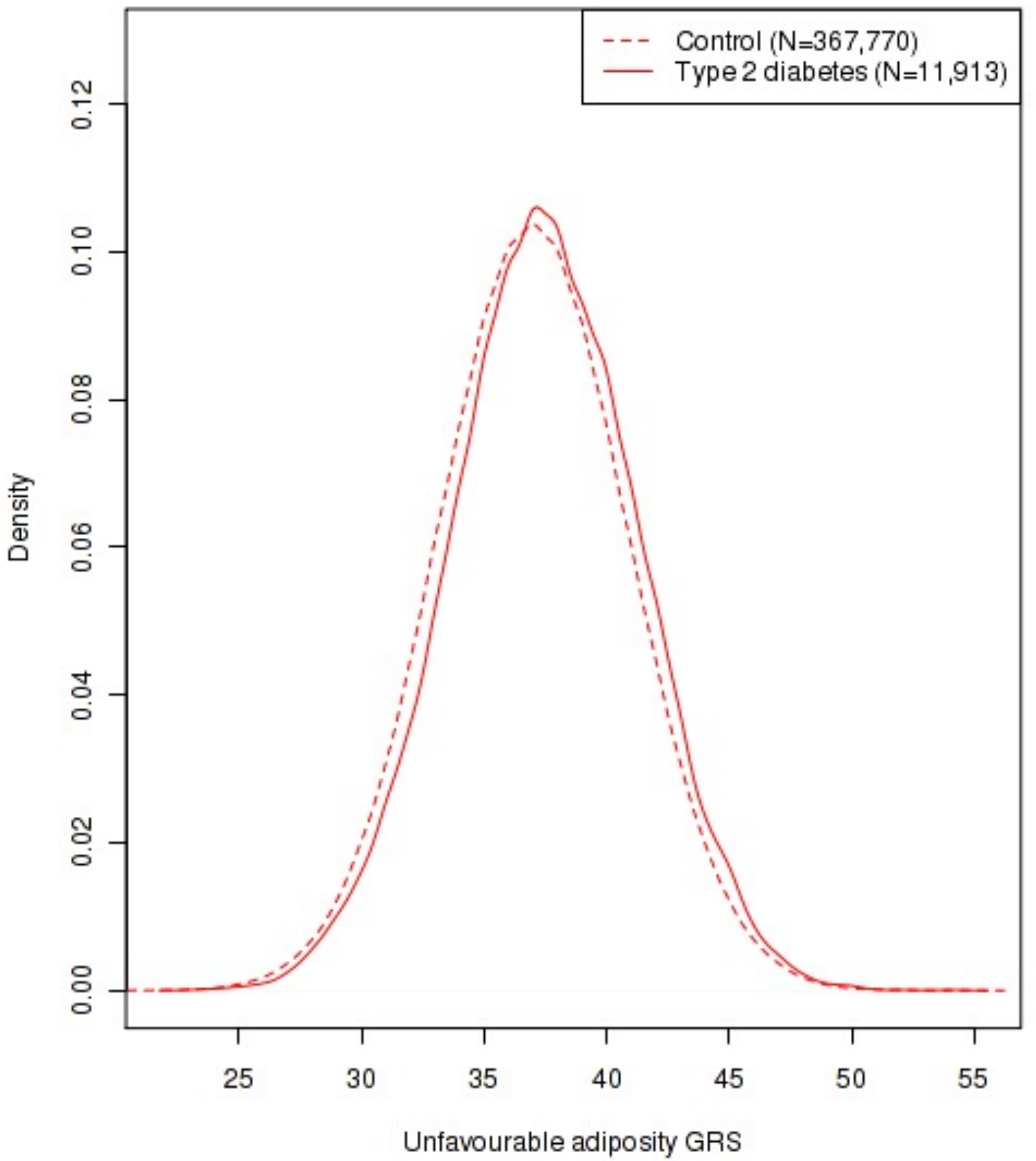




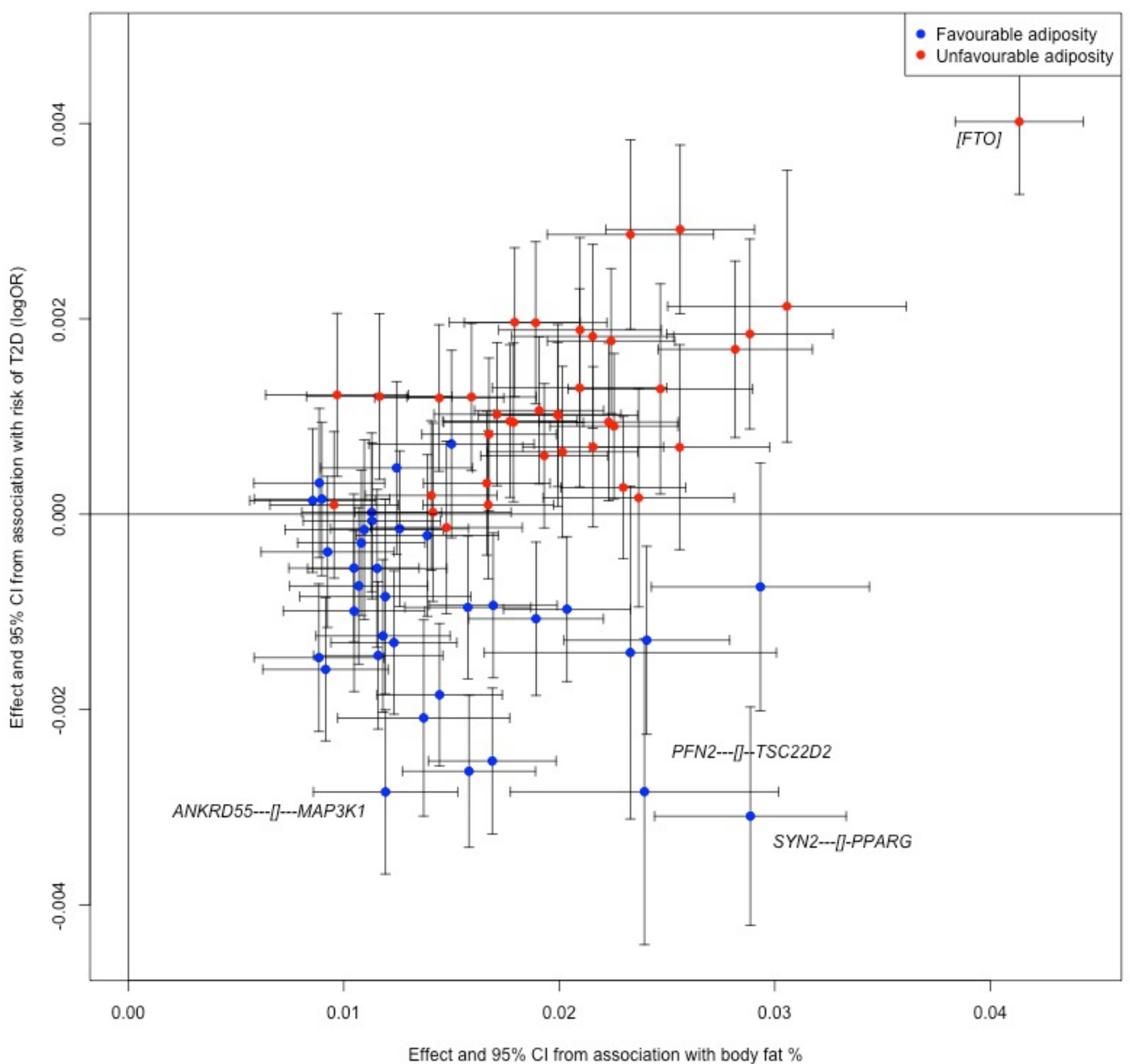


UK Biobank liver fat

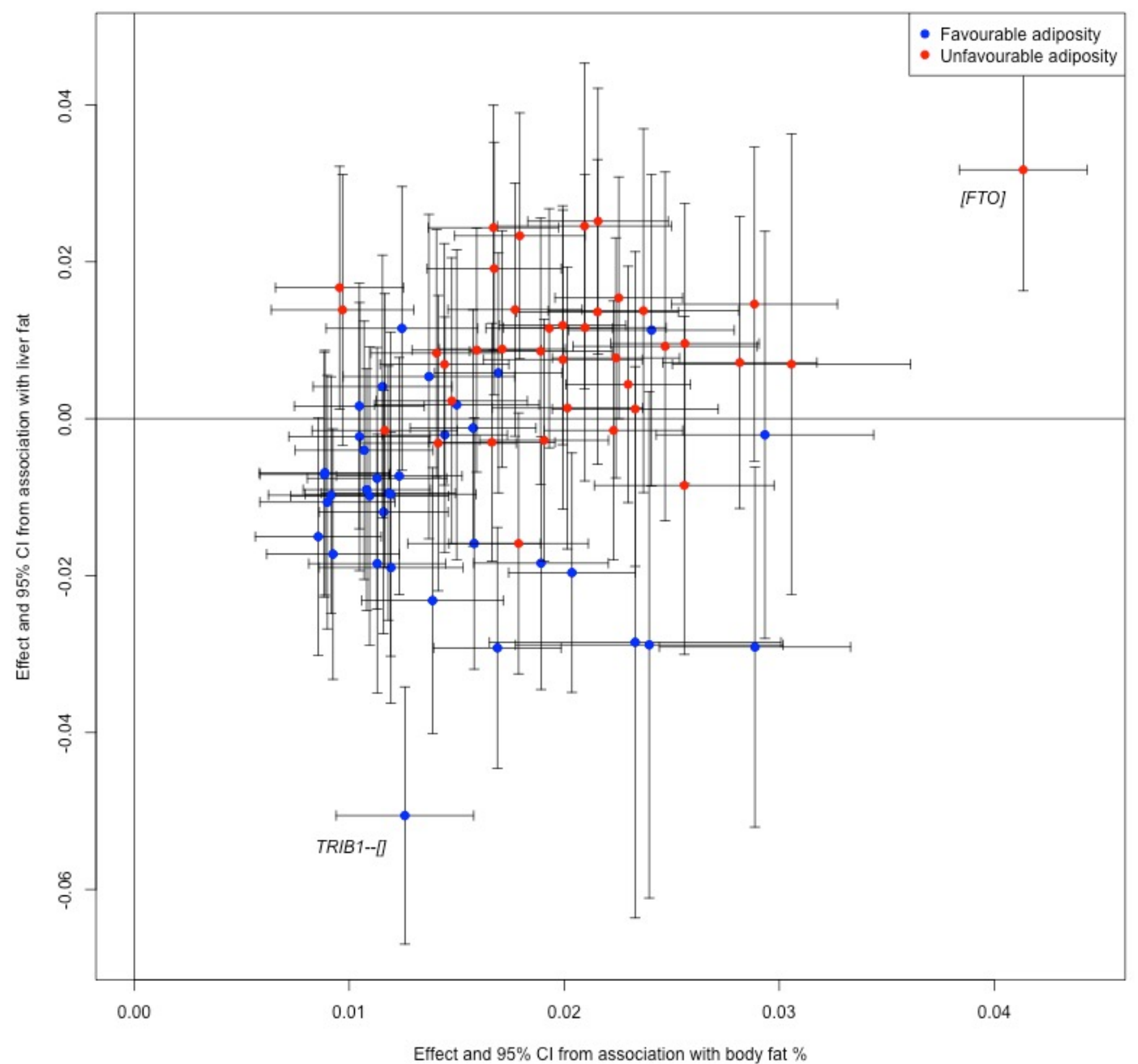


UK Biobank pancreas fat

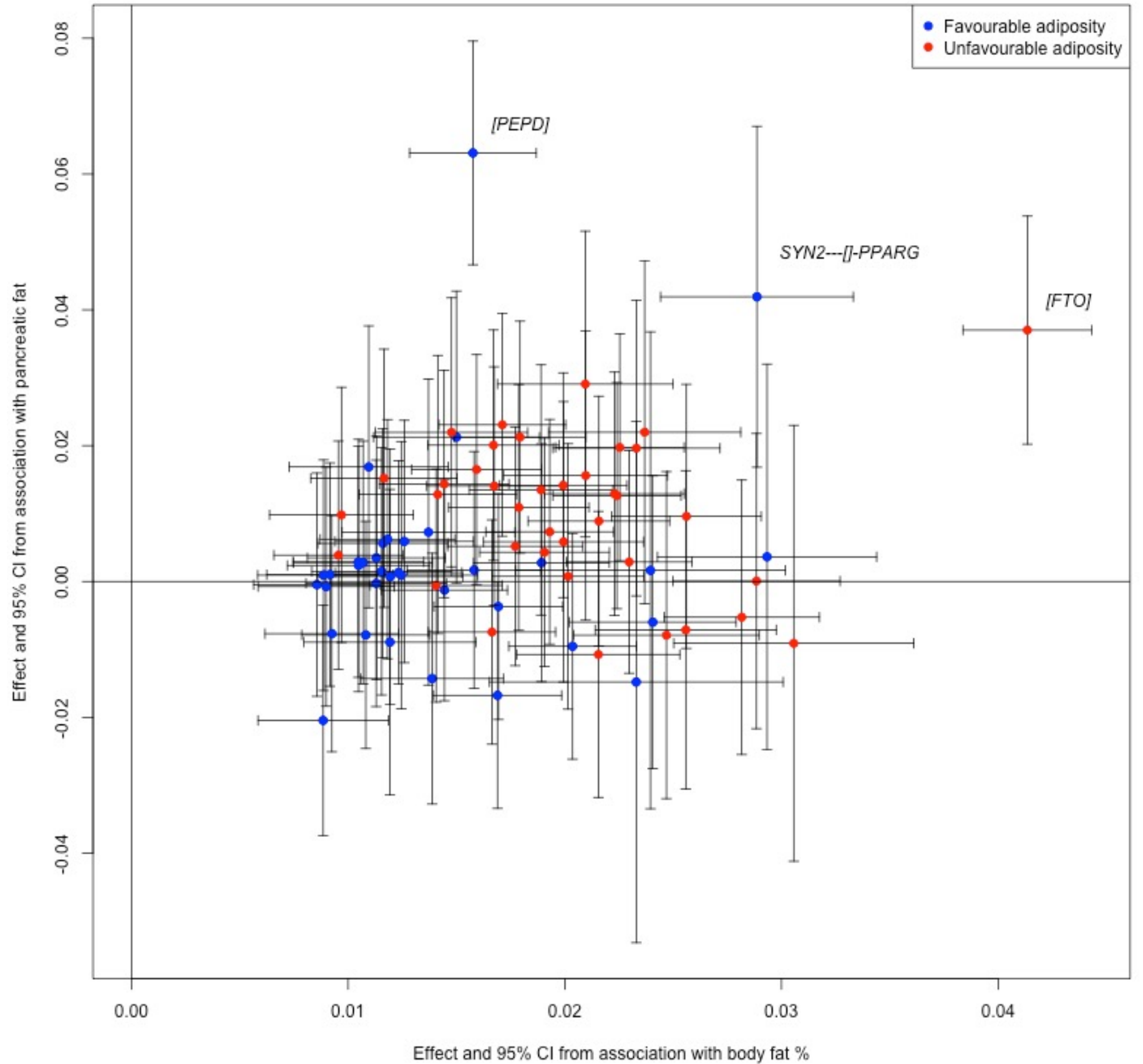


rs4684847 - SYN2---D-PPARG

SF 8a

Body fat \%

BMI

HDL

SHBG

Triglycerides

ALT

AST

CRP

ASAT

VAT

VATSAT

Liver fat

Pancreatic fat

Liver size

Pancreas size

T2D (logOR)

Heart disease (logOR)

Hypertension (logOR)
Effect

0.03

0.01

0.02

0.03

$-0.03$

$-0.03$

$-0.02$

5e-04

0.04

$-0.008$

$-0.05$

$-0.03$

0.04

3e-05

$-0.03$

$-0.006$

1e-04

$-0.004$
P value

$7.5 \mathrm{e}-24$

2e-06

$5.9 \mathrm{e}-12$

$6.2 \mathrm{e}-33$

1.1e-18

$2.7 \mathrm{e}-31$

1.1e-16

0.74

3.9e-05

0.42

4. $4 \mathrm{e}-10$

0.013

0.0011

1

0.0033

7.2e-14

0.89

0.0024
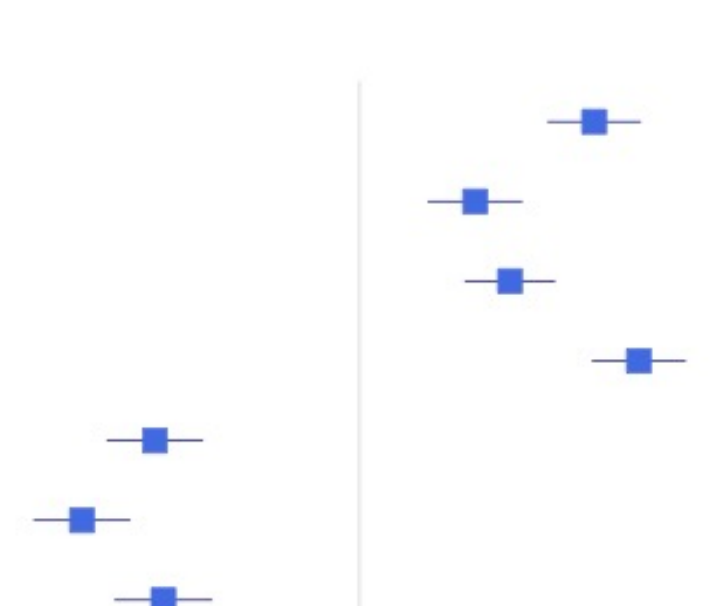

$\rightarrow$
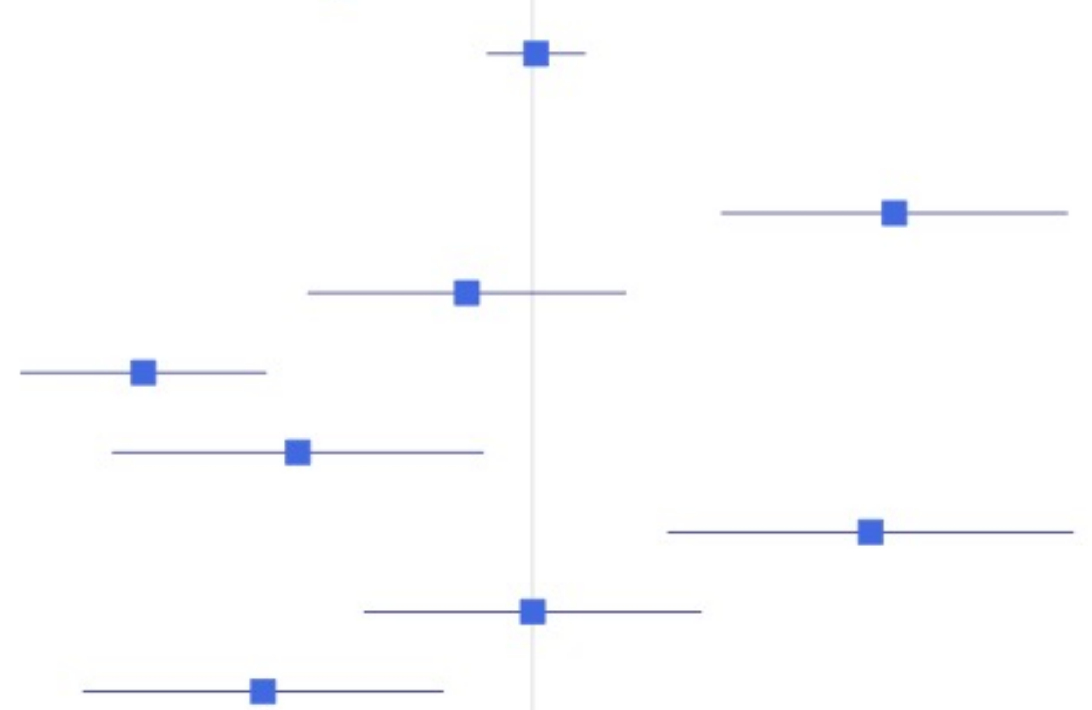

?

Null effect 
rs12130231 - LYPLAL1---[---SLC30A10

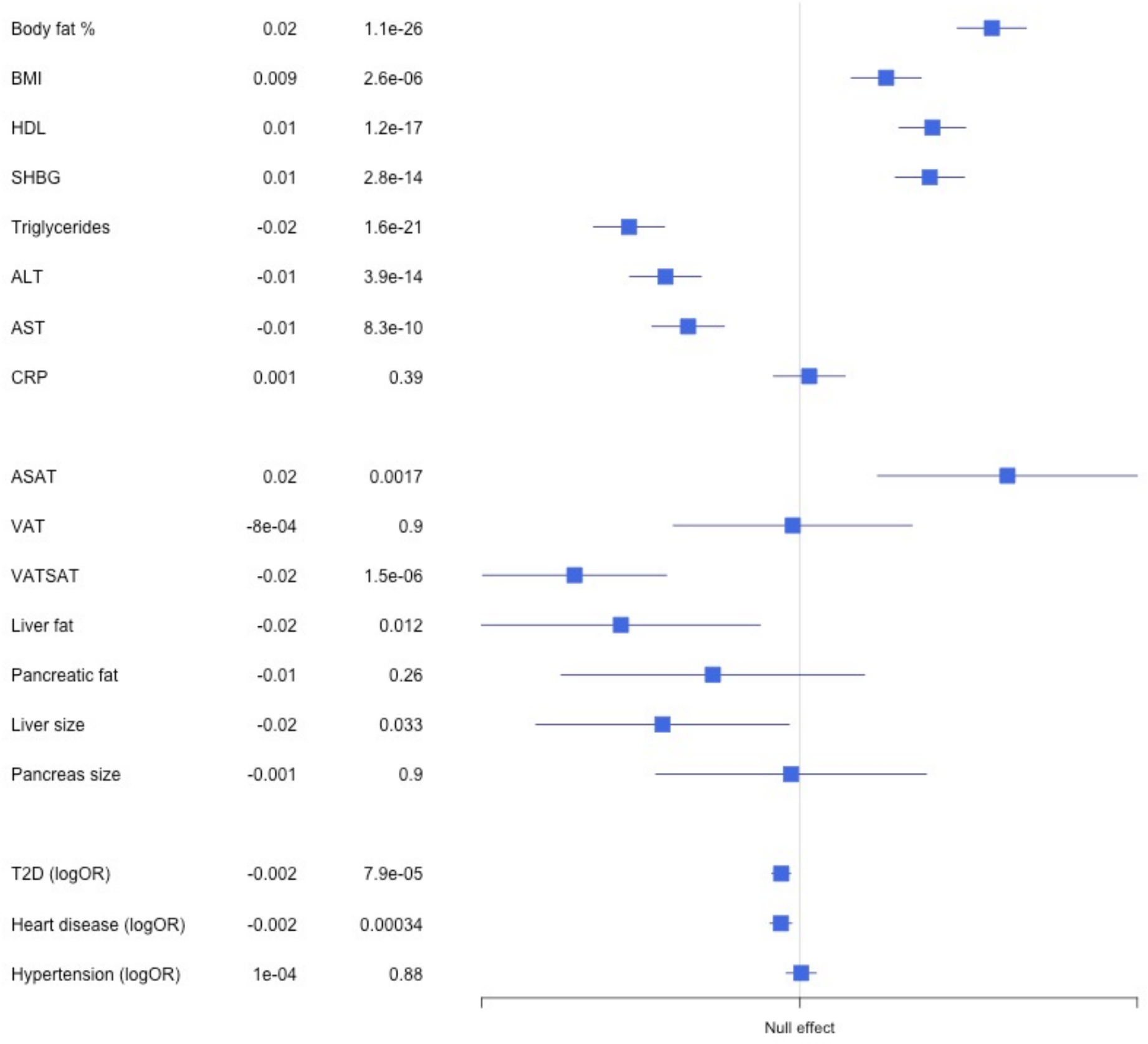




\section{SF 8c}

Body fat $\%$

BMI

HDL

SHBG

Triglycerides

ALT

AST

CRP

ASAT

VAT

VATSAT

Liver fat

Pancreatic fat

Liver size

Pancreas size

T2D (logOR)

Heart disease (logoR)

Hypertension (logOR) rs11664106 - SMCHD1--[DEMILIN2

Effect $P$ value

$0.009 \quad 2.8 \mathrm{e}-06$

$0.007 \quad 0.0014$

$0.009 \quad 1.1 \mathrm{e}-05$

$0.01 \quad 1.9 \mathrm{e}-09$

$-0.01 \quad 1.2 \mathrm{e}-09$

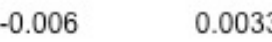

$-0.002-0.3$

0.002

0.29

$0.01 \quad 0.052$

$\begin{array}{ll}-0.009 & 0.18\end{array}$

$-0.01 \quad 0.0055$

$\begin{array}{ll}-0.02 & 0.034\end{array}$

$\begin{array}{ll}-0.008 & 0.39\end{array}$

$\begin{array}{ll}-0.01 & 0.065\end{array}$

$\begin{array}{ll}-0.006 & 0.48\end{array}$

$-4 \mathrm{e}-04 \quad 0.44$

$3 e-04 \quad 0.64$

$-0.002-0.038$
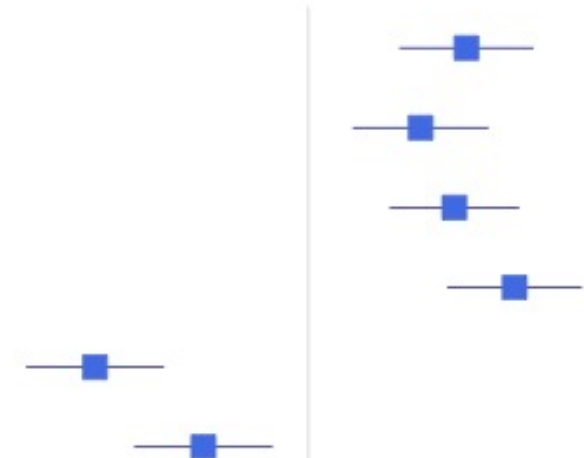

$\longrightarrow$

$-$

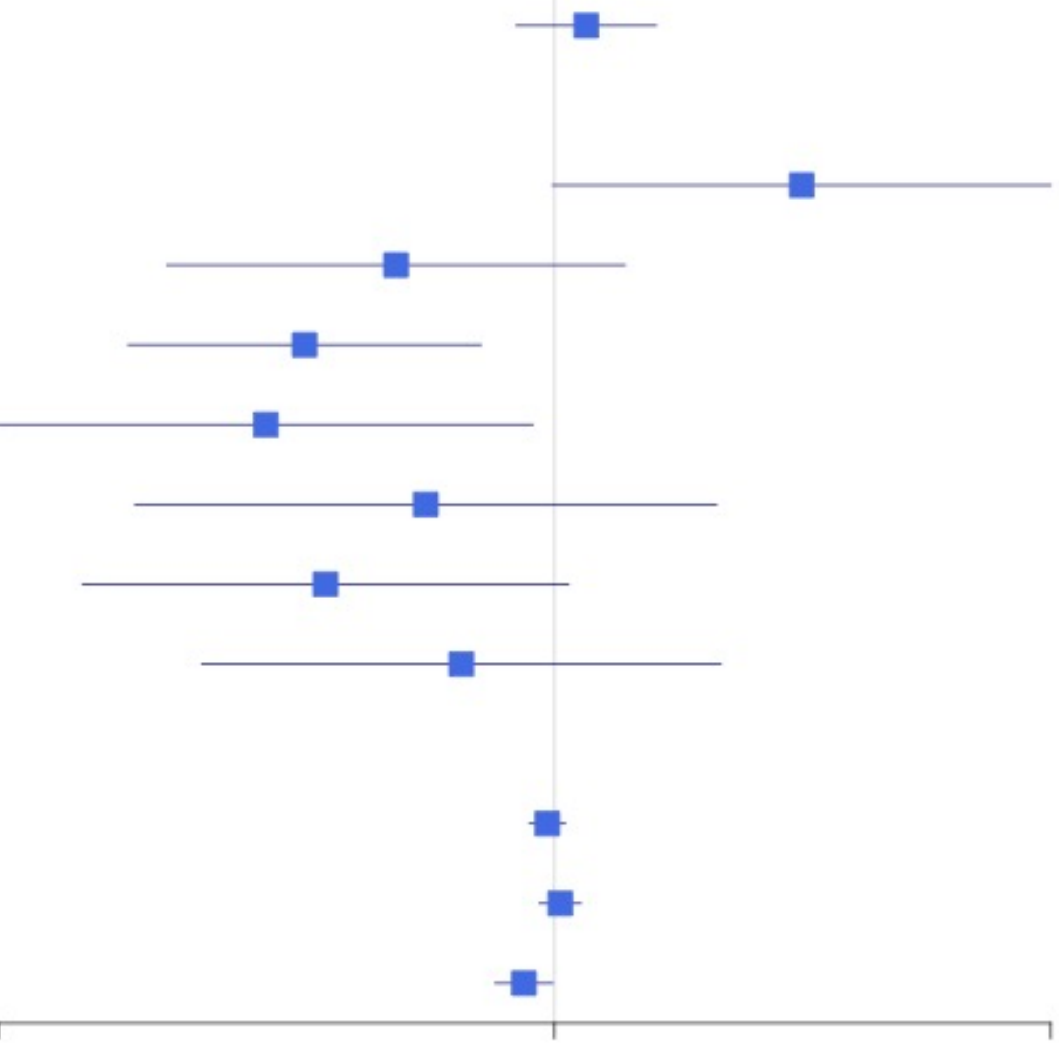

Null effect 


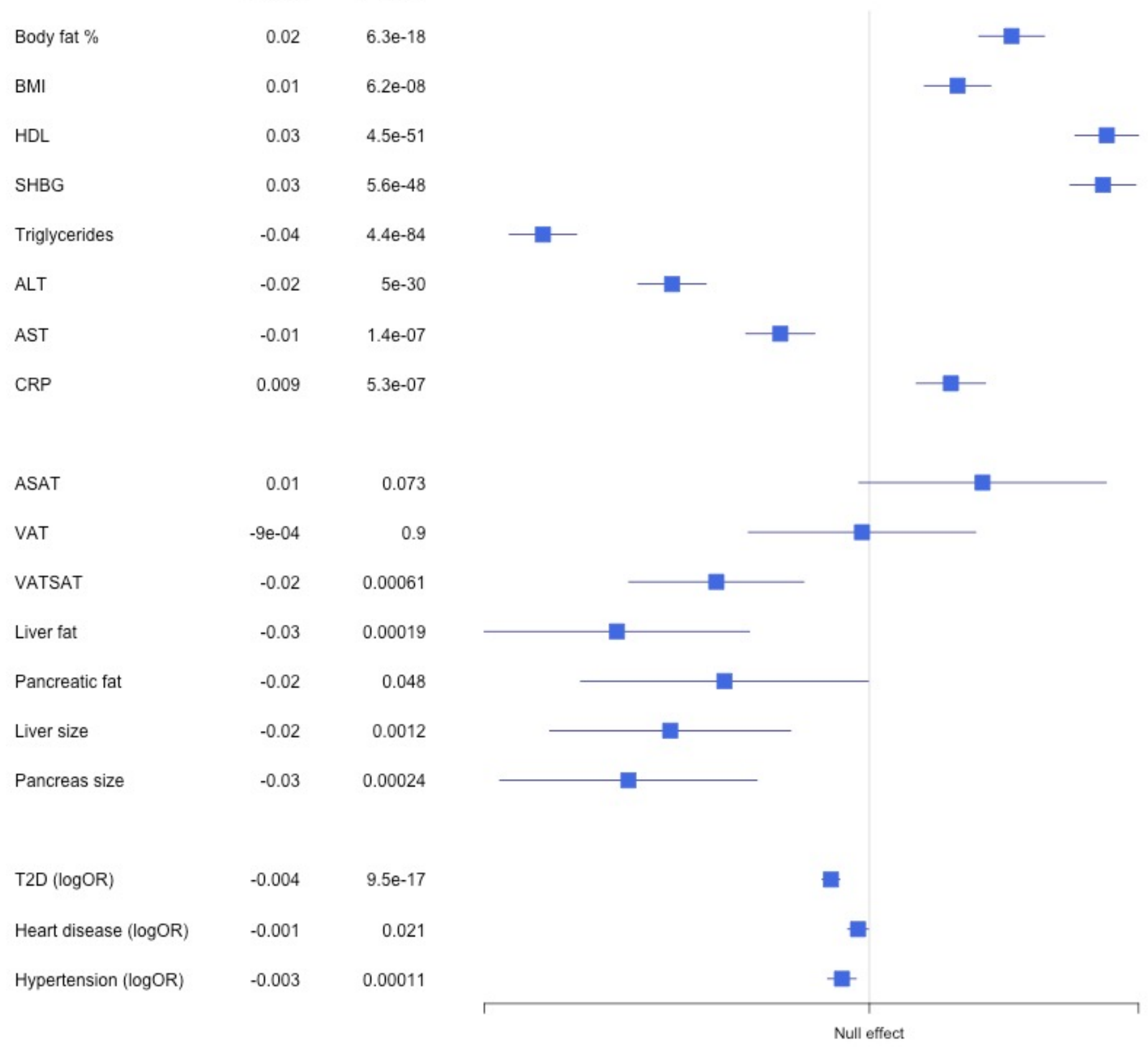




\section{SF 8e}

Body fat \%

BMI

HDL

SHBG

Triglycerides

ALT

AST

CRP

ASAT

VAT

VATSAT

Liver fat

Pancreatic fat

Liver volume

Pancreas volume

T2D (logOR)

Heart disease $(\log O R)$

Hypertension (logOR)
Effect

0.01

0.003

0.04

0.03

$-0.04$

$-0.03$

$-0.02$

$-0.003$

0.02

0.006

$-0.03$

$-0.02$

0.002

$-0.01$

$-0.02$

$-0.005$

$-0.002$

$-0.003$

rs2943653 - NYAP2---[---IRS1

$P$ value

3.2e-11

0.19

$1.8 \mathrm{e}-100$

$2.4 \mathrm{e}-39$

$2.9 \mathrm{e}-76$

$4 e-34$

$4.4 \mathrm{e}-17$

0.086

0.0024

0.4

$1.7 \mathrm{e}-07$

0.05

0.85

0.13

0.0054
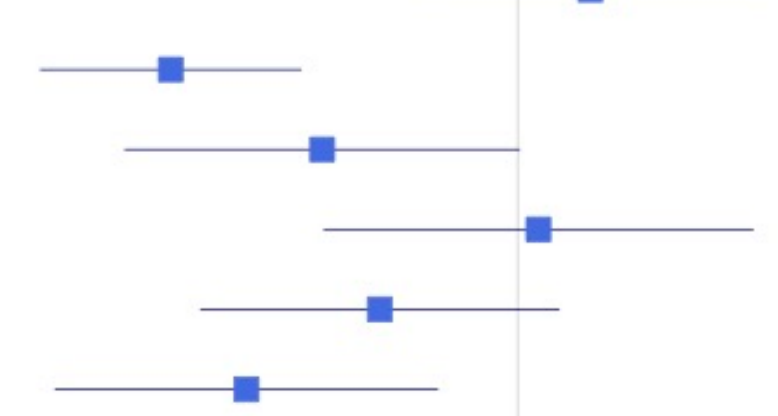

$2.2 \mathrm{e}-22$

0.0011

0.0022

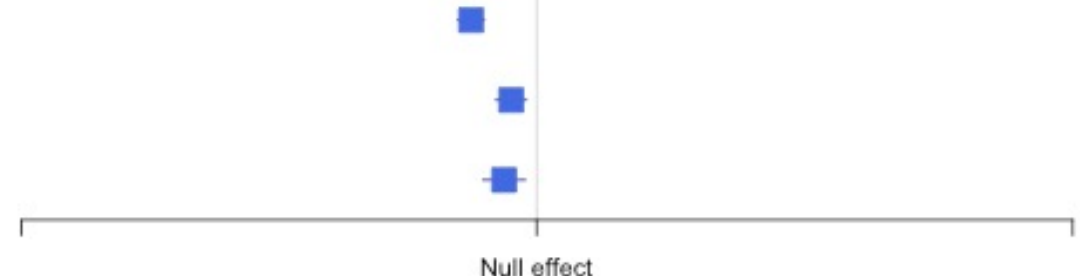




\section{SF $8 f$}

Body fat \%

BMI

HDL

SHBG

Triglycerides

ALT

AST

CRP

ASAT

VAT

VATSAT

Liver fat

Pancreatic fat

Liver size

Pancreas size

T2D (logOR)

Heart disease $(\log O R)$

Hypertension (logOR) rs30351 - ANKRD55---[]--MAP3K1

\section{Effect}

0.01

$-6 e-04$

0.03

0.03

$-0.03$

$-0.02$

$-4 \mathrm{e}-04$

0.01

$0.02 \quad 0.053$

0.05

0.03

$-0.02$

$7 \mathrm{e}-04$

$-0.01$

$-0.03$

$-0.004$

$-0.002$

$-0.004$

\section{$P$ value}

$8.4 \mathrm{e}-07$

0.74

$1.7 \mathrm{e}-38$

$5.1 \mathrm{e}-42$

$9.3 e-44$

$6.9 \mathrm{e}-16$

0.7

$2.2 \mathrm{e}-10$

4.2e-06

0.031

0.94

0.073

0.00017

$1.6 \mathrm{e}-09$

0.016

5.1e-06
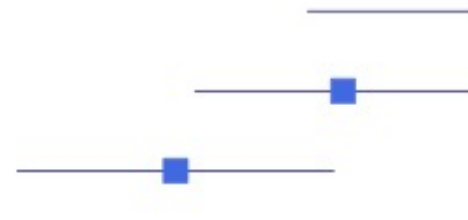

[

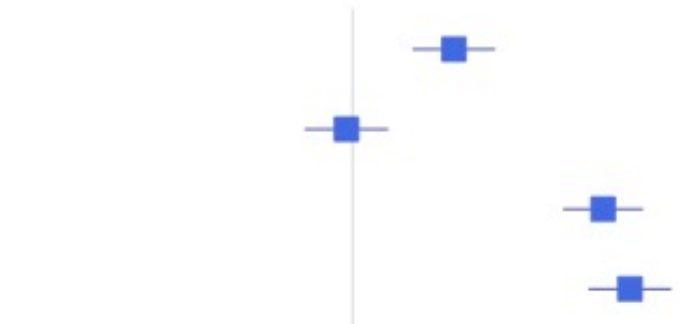

-

-

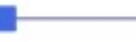

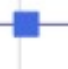

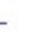


rs4450871 - MSX1---[--CYTL1

\section{SF 8g}

Body fat $\%$

BMI

$\mathrm{HDL}$

SHBG

Triglycerides

ALT

AST

CRP

ASAT

VAT

VATSAT

Liver fat

Pancreatic fat

Liver volume

Pancreas volume

T2D (logOR)

Heart disease $(\log O R)$

Hypertension (logOR)
Effect

0.009

0.007

0.008

0.01

$-0.01$

$-0.009$

$-0.005$

0.005

0.01

$-1 \mathrm{e}-04$

$-0.01$

$-0.02$

$-5 \mathrm{e}-04$

$-0.009$

$-0.02$

$-6 e-04$

$-8 \mathrm{e}-04$

$-2 \mathrm{e}-04$

\section{$P$ value}

$3.4 \mathrm{e}-06$

0.00035

5.4e- 07

$2.6 \mathrm{e}-08$

$9.6 \mathrm{e}-13$

9e-07

0.0092

0.055

0.14

0.99

0.043

0.05

0.96

0.18

0.0068

0.2

0.19

0.87
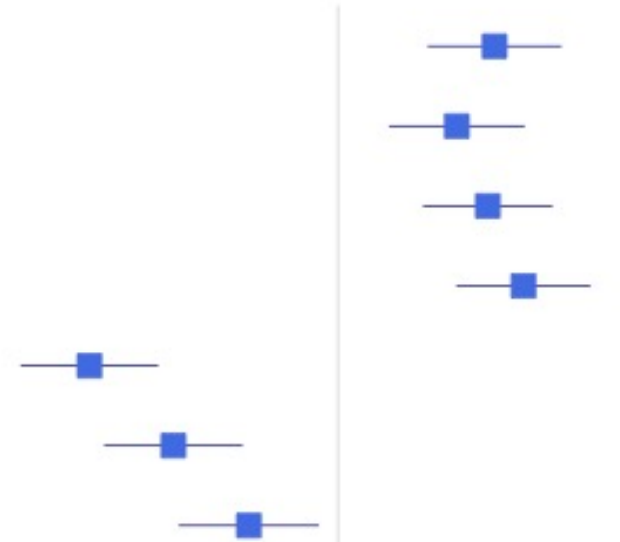

$+$
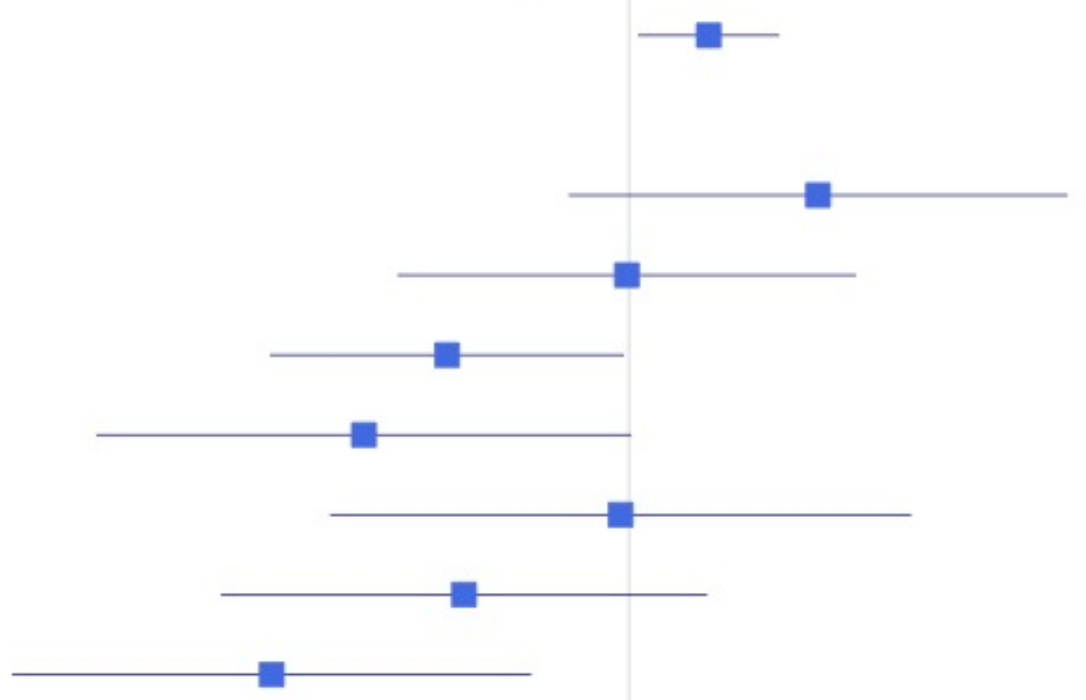

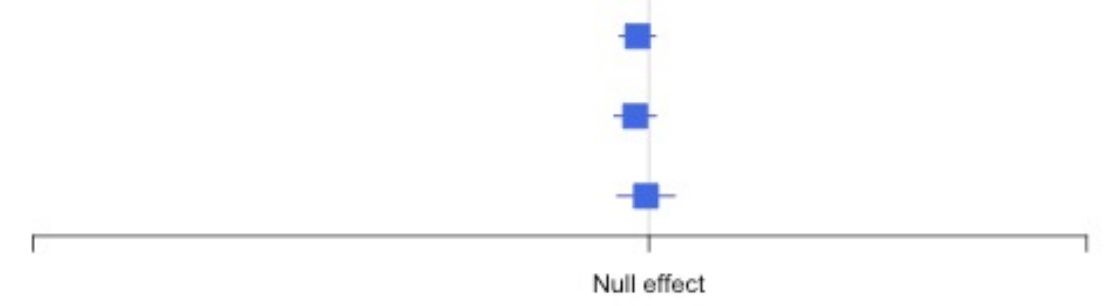




\section{SF $8 h$}

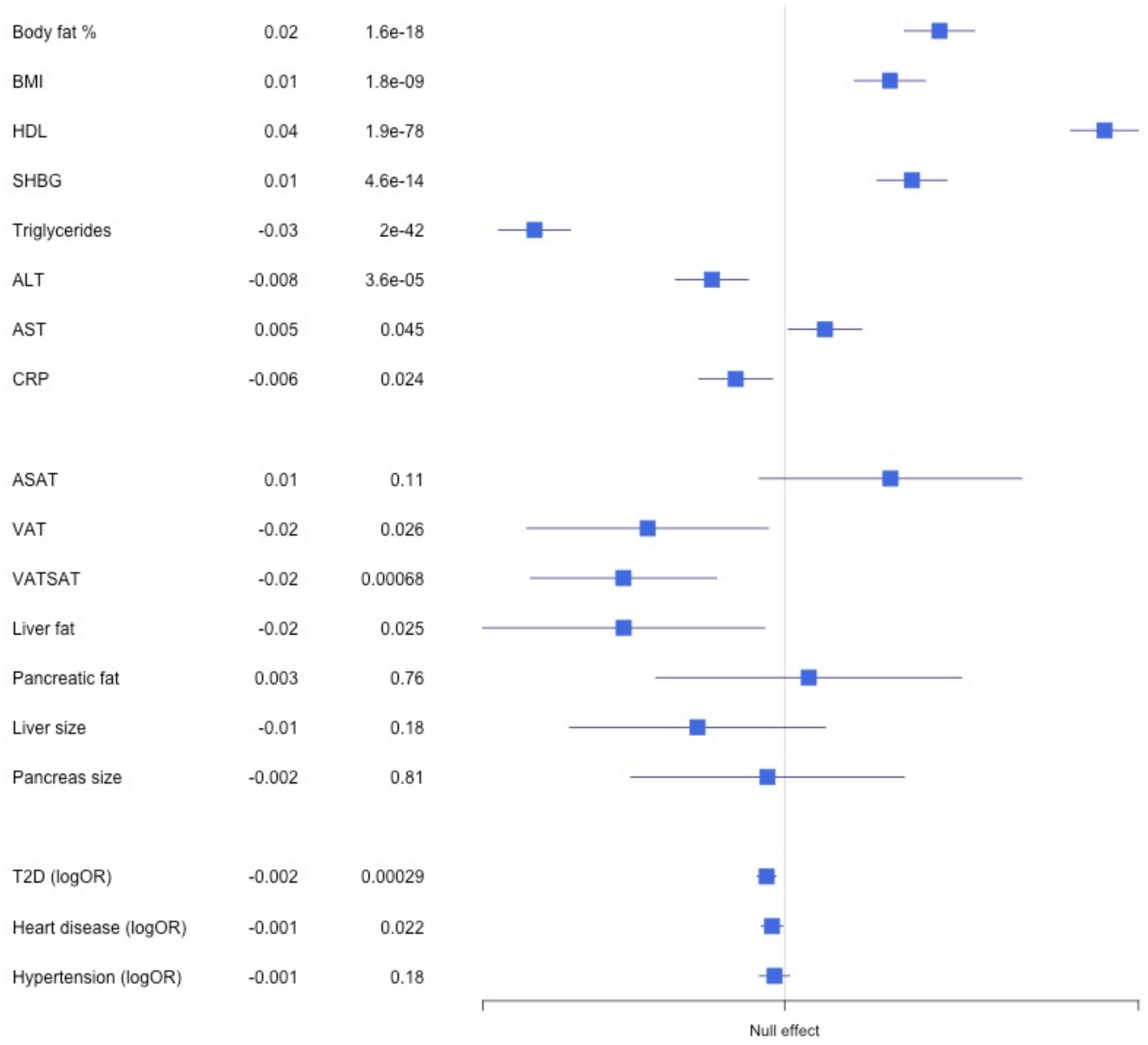




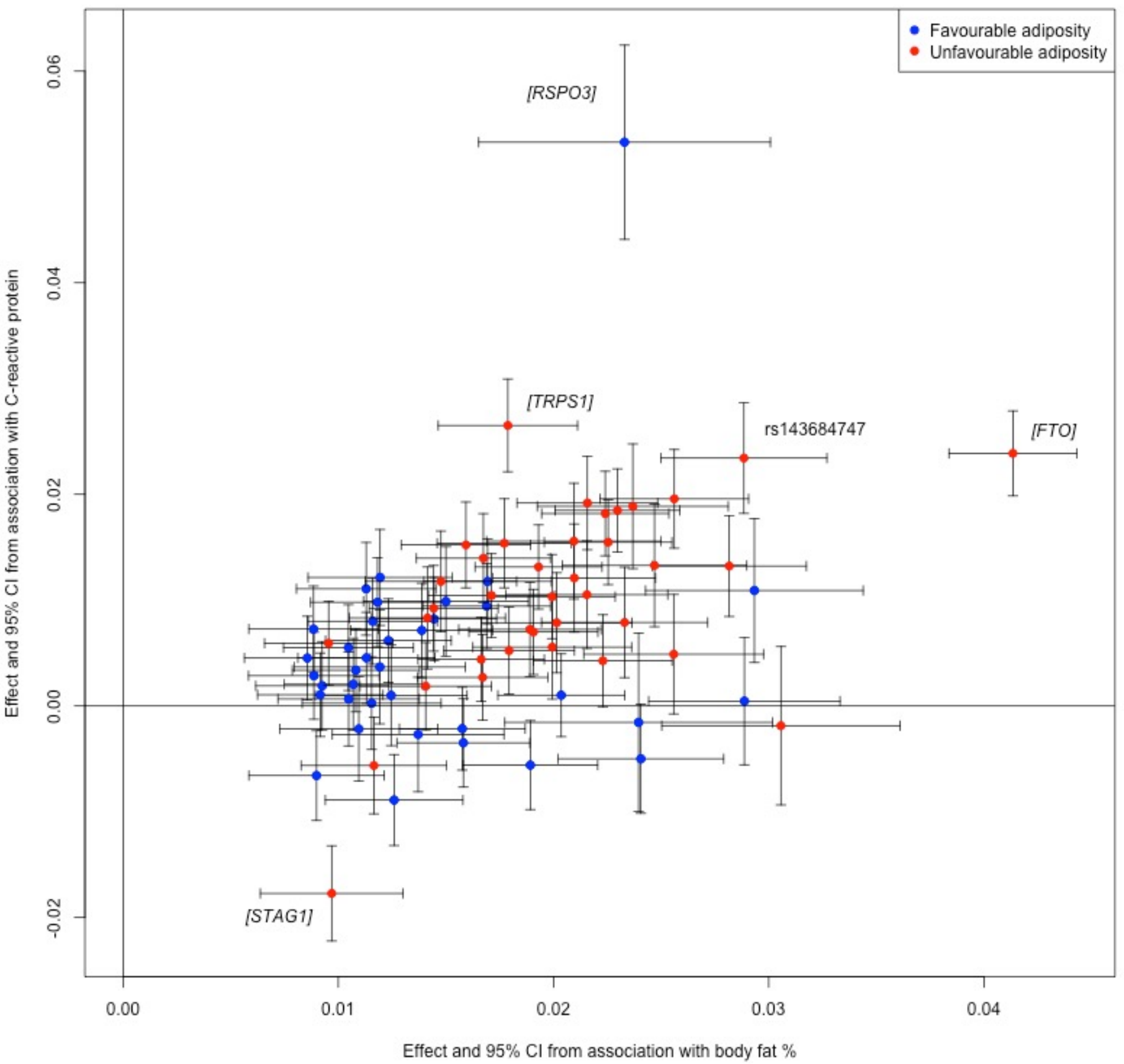




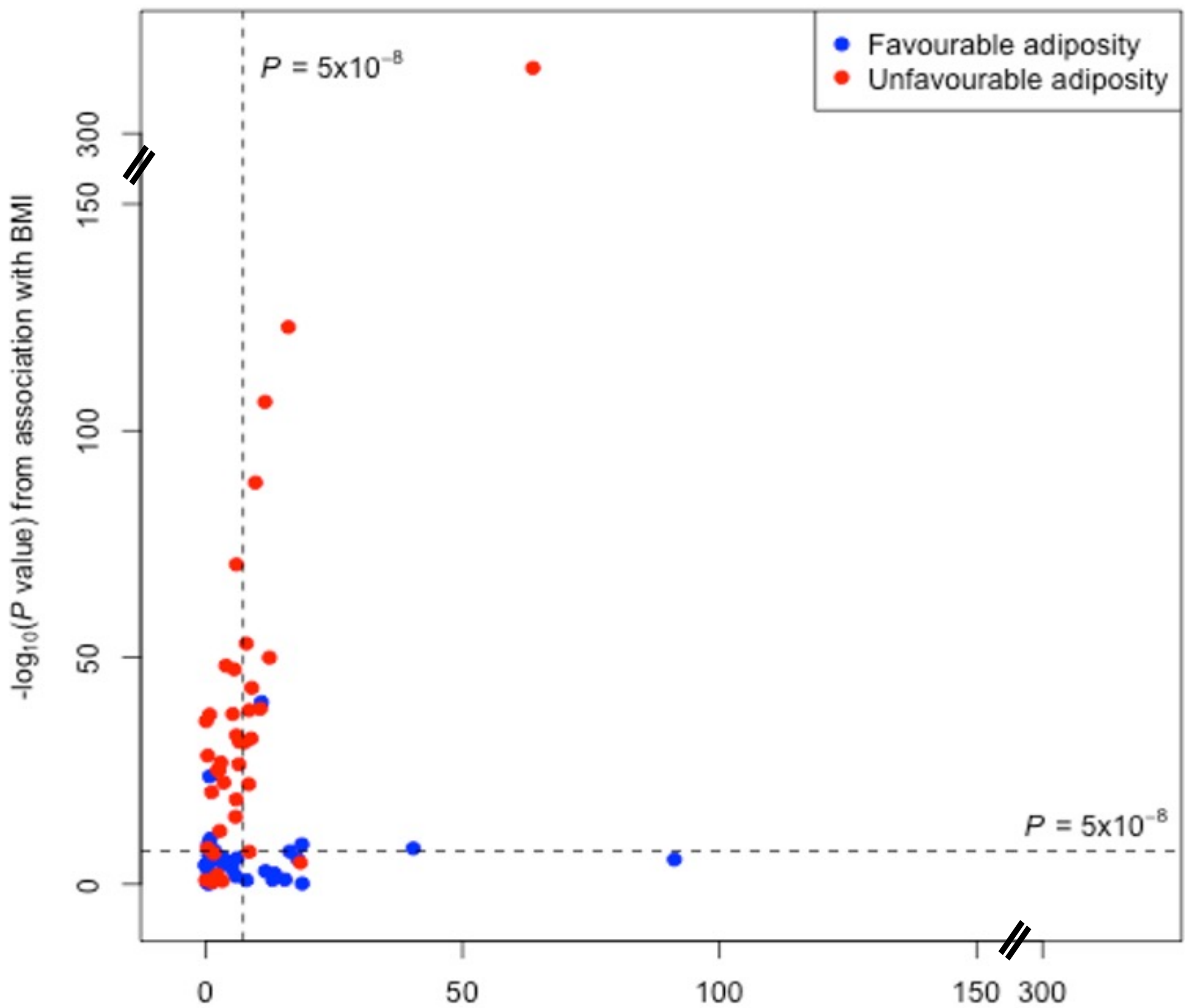

$-\log _{10}(P$ value $)$ from association with waist-hip-ratio 


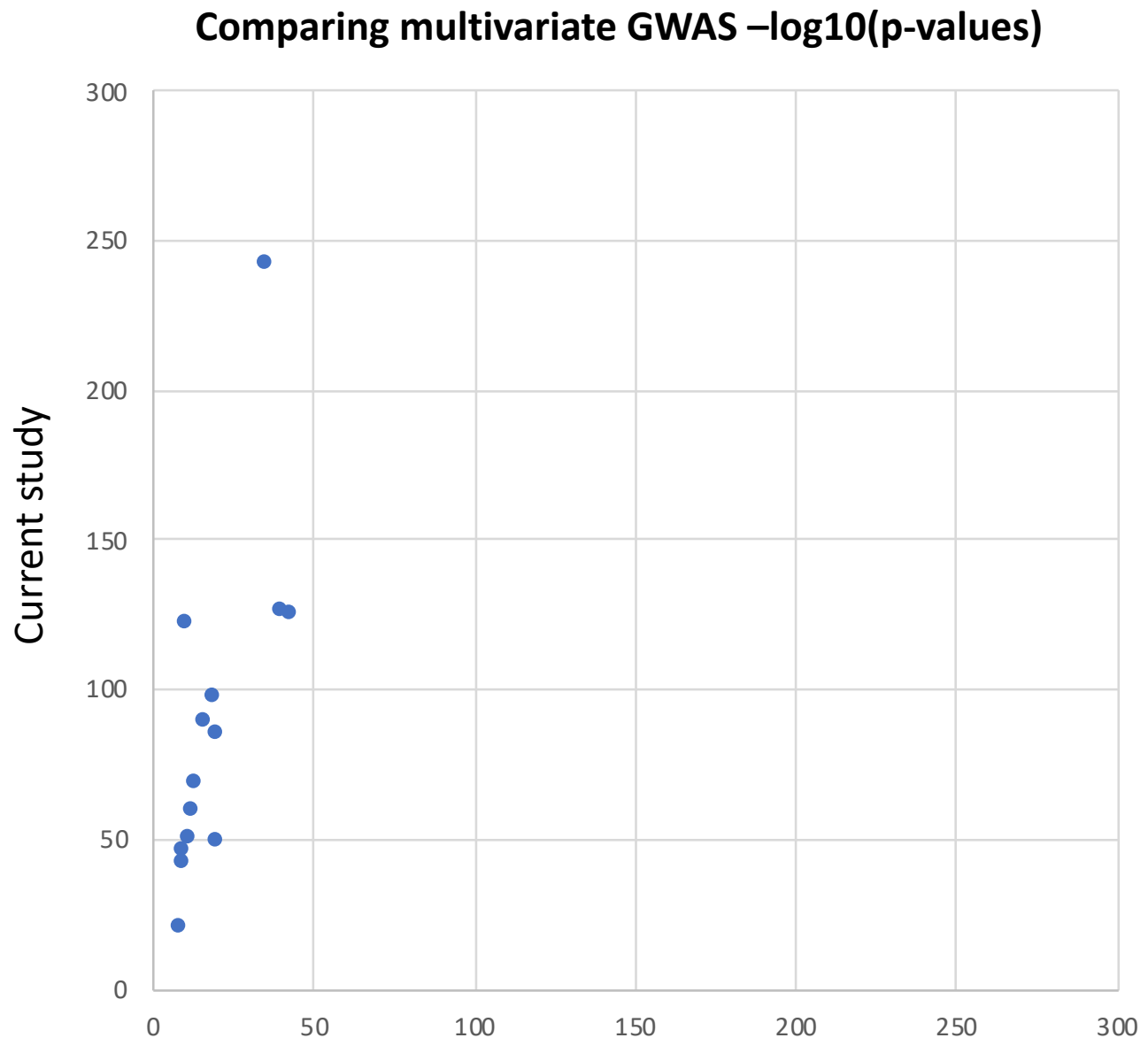

From Ji et al. Diabetes, 2019 